\title{
Volatile Organic Compounds (VOCs) Produced By The Rhizosphere Actinobacteria Streptomyces Sp. and Amycolatopsis Sp. Strains and Their Antifungal Activity Against Colletotrichum Musae
}

Maria Vieira de Brito

Universidade Federal do Ceará

Wéverson L. Fonseca

Universidade Federal do Piauí

Jair Mafezoli

Universidade Federal do Ceará

Francisco Geraldo Barbosa

Universidade Federal do Ceará

Fátima Miranda Nunes

Universidade Federal do Ceará

Marcos Carlos de Mattos

Universidade Federal do Ceará

João E. A. dos Santos

Universidade Federal do Ceará

Francisca S. A. Araujo

EMBRAPA Agroindústria Tropical

Regimara F. B. S. Vieira

EMBRAPA Agroindústria Tropical

Hilton C. R. Magalhães

EMBRAPA Agroindústria Tropical

Celli R. Muniz

EMBRAPA Agroindústria Tropical

Deborah S. Garruti

EMBRAPA Agroindústria Tropical

Marcio A. Ootani

Cidade Universitária

José M. S. Netto

Universidade Federal de Pernambuco

Licarion Pinto

Universidade Federal de Pernambuco 


\section{Francisco M.P. Viana}

EMBRAPA Agroindústria Tropical

Maria da Conceicao Ferreira de Oliveira ( $\square$ mcfo@ufc.br)

Federal University of Ceara: Universidade Federal do Ceara https://orcid.org/0000-0002-7445-2347

\section{Research Article}

Keywords: VOCs, HS-SPME-GCMS, Amycolatopsis, Streptomyces, Colletotrichum musae, Chemometrics

Posted Date: May 13th, 2021

DOl: https://doi.org/10.21203/rs.3.rs-509649/v1

License: (c) (1) This work is licensed under a Creative Commons Attribution 4.0 International License.

Read Full License 


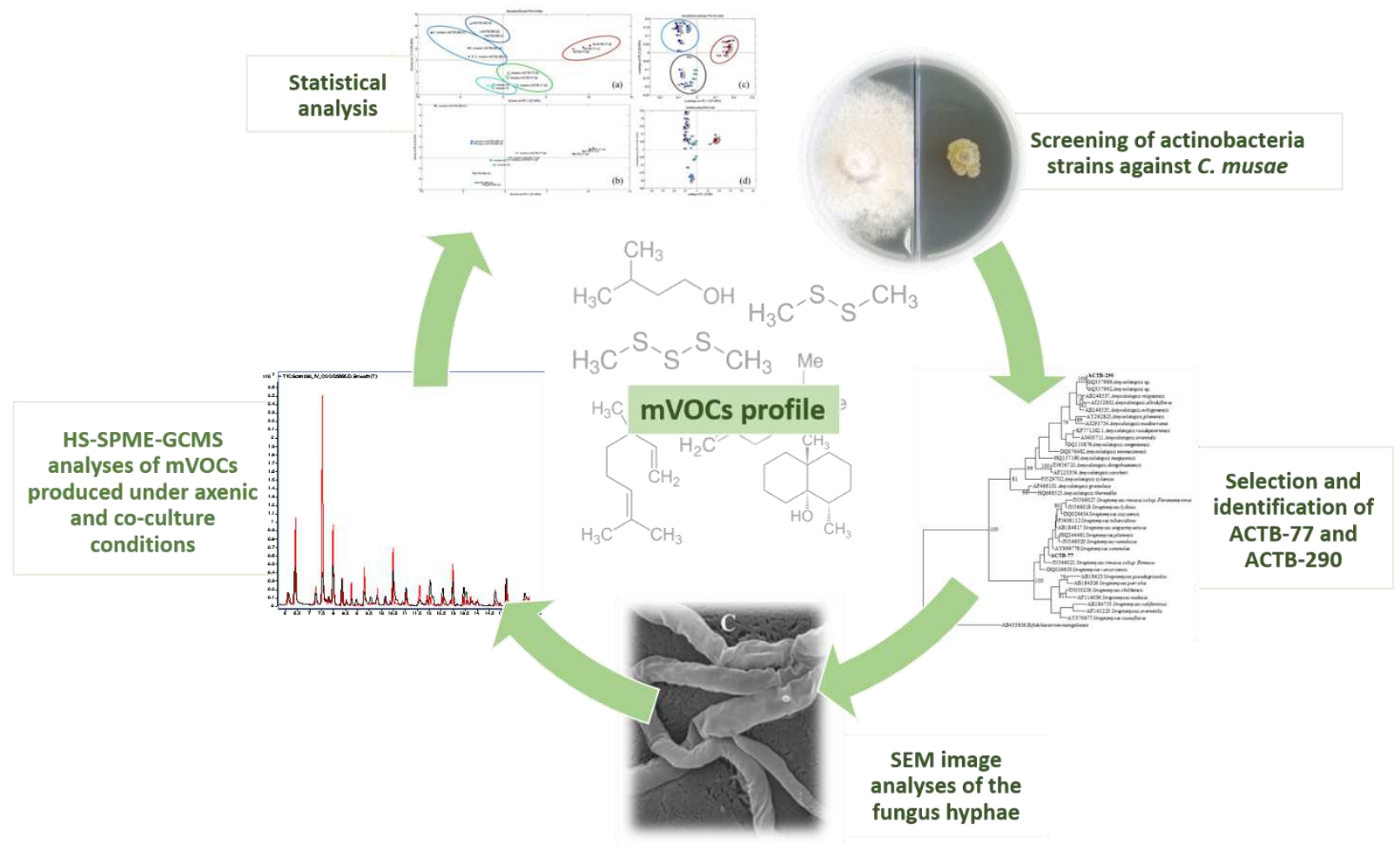


5 Volatile organic compounds (VOCs) produced by the rhizosphere actinobacteria

6 Streptomyces sp. and Amycolatopsis sp. strains and their antifungal activity against

7

8

\section{Colletotrichum musae ${ }^{\dagger}$}

Maria V. de Brito ${ }^{1}$-Wéverson L. Fonseca ${ }^{2}$ Jair Mafezoli $^{1} \cdot$ Francisco G. Barbosa $^{1} \cdot$ Fátima M. Nunes $^{1}$ - Marcos C. de Mattos $^{1}$ • João E. A. dos Santos • Francisca S. A. Araujo $^{3}$ - Regimara F. B. S. Vieira ${ }^{3}$ Hilton C. R. Magalhães ${ }^{3} \cdot$ Celli R. Muniz ${ }^{3} \cdot$ Deborah S. Garruti $^{3} \cdot$ Marcio A. Ootani $^{4} \cdot$ José M. S. Netto $^{5}$ - Licarion Pinto $^{5}$ • Francisco M. P. Viana ${ }^{3, \dagger} \bullet$ Maria C. F. Oliveira ${ }^{1, *}$

${ }^{1}$ Departamento de Química Orgânica e Inorgânica, Centro de Ciências, Universidade Federal do Ceará, Campus do Pici, Bl. 940, Fortaleza-CE, 60.440-593, Brazil.

${ }^{2}$ Colégio Técnico de Bom Jesus-CTBJ, Universidade Federal do Piauí, BR 135, km 3 - Planalto Horizonte, Bom Jesus-PI, 64900-000, Brazil.

${ }^{3}$ EMBRAPA Agroindústria Tropical, Rua Pernambuco 2270, Pici, Fortaleza-CE, 60511-110, Brazil.

4 Centro de Tecnologias Estrategicas do Nordeste-CETENE, Av. Prof. Luiz Freire - lado par, Cidade Universitária, Recife-PE, 50.740-540, Brazil.

${ }^{5}$ Universidade Federal de Pernambuco, Laboratório de Metabolômica e Quimiometria, Av. Jornalista Anibal Fernandes s/n, Cidade Universitária, Recife-PE, 50.740-560, Brazil.

\section{Abstract}

Herein is reported the study on the antifungal potential of VOCs produced by actinobacterial strains from the rhizosphere of Caatinga plants. Twenty strains were screened for their ability to produce VOCs with growth inhibition (GI) potential against the phytopathogen fungus Colletotrichum musae. The most active VOCs were produced by Streptomyces sp. (ACTB-77) and Amycolatopsis sp. (ACTB-290). The effect of VOCs from ACTB-77 and ACTB-290 on the morphological structure of the fungus hyphae was investigated by SEM and revealed significant deformation of the fungal cell filaments, especially in the case of ACTB-290. VOCs produced by these two actinobacteria strains and the fungus under axenic and co-culture conditions were investigated using HS-SPME-GCMS combined approaches, and all data were analyzed by statistical methods. Most of the mVOCs were identified, representing $92-100 \%$ of total peaks areas. Linalool $(38.53 \pm 5.19 \%)$ and geosmin $(17.25 \pm 2.55 \%)$ were the major VOCs constituents from ACTB-77, while dimethyl disulfide (48.49 $\pm 3.48 \%)$ and dimethyl trisulfide $(21.14 \pm 3.50 \%)$ were the major VOCs compounds produced by ACTB-290. Statistical analyses of data from co-cultures showed that the VOCs profile of the experiment involving ACTB-77 was more influenced by the fungal VOCs while the experiment with ACTB-290 was more influence by compounds from the actinobacteria.

\section{Keywords}

VOCs $\bullet$ HS-SPME-GCMS $\bullet$ Amycolatopsis $\bullet$ Streptomyces $\bullet$ Colletotrichum musae $\bullet$ Chemometrics

\footnotetext{
${ }^{*}$ Corresponding author: M. C. F. Oliveira (mcfo@ufc.br)

${ }^{\dagger}$ In Memoriam. This paper is dedicated to Dr. Francisco Marto Pinto Viana, Brazilian phytopathologist who motivated us to develop this research and passed away during the manuscript preparation.
} 


\section{Declarations}

\section{Funding}

This study was financed in part by the Coordenação de Aperfeiçoamento de Pessoal de Nível Superior - Brasil (CAPES) Finance Code 001 (PROEX 23038.000509/2020-82), Conselho Nacional de Desenvolvimento Científico e Tecnológico (CNPq) - (Process: 310881/2020-0 and Process: 306043/2018-1) and Fundação de Amparo a Ciência e Tecnologia do Estado de Pernambuco (FACEPE 14/2019 - INOVA IAM; Process: APQ-0437-1.06/19).

\section{Conflicts of interest/Competing interests}

The authors declare no Conflicts of interest/Competing interests

\section{Availability of data and material (data transparency)}

All data presented in the manuscript are original and were obtained by the authors, including figures and tables.

Code availability (software application or custom code)

Not applicable

Authors' contributions

All authors contributed to the study conception and design. Material preparation, data collection and analysis were performed as follows:

Maria V. de Brito, Wéverson L. Fonseca, Jair Mafezoli, Francisco G. Barbosa, Fátima M. Nunes, Marcos C. de Mattos, João E. A. dos Santos and Maria C. F. Oliveira - Culture of the microorganisms, VOCs extractions and analyses (HS-SPMEGCMS), actinobacteria molecular identification, manuscript writing and revision.

Francisca S. A. Araujo, Regimara F. B. S. Vieira, Hilton C. R. Magalhães, Celli R. Muniz and Deborah S. Garruti - SEM image analysis, GCMS equipment operation and microorganisms preservation.

Marcio A. Ootani and Francisco M. P. Viana - Isolation of actinobateria strains from rhizosphere associated to Caatinga plants and Colletotrichum musase from infected plant.

José M. S. Netto and Licarion Pinto - Statistical analyses.

The first draft of the manuscript was written by Maria C. F. Oliveira (corresponding author) and all authors commented on previous versions of the manuscript. All authors read and approved the final manuscript.

\section{Ethics approval}

Not applicable 


\section{Introduction}

Microorganisms present a sophisticated metabolism that is responsible for the production of a myriad of metabolites with diverse molecular structures. Many of the microbial producing compounds, especially those from the secondary metabolism, are known for their biotechnological potential [1,2]. Besides producing non-volatile chemical structures, microorganisms can also provide volatile organic compounds (VOCs), also referred as microbial VOCs (mVOCs), which play important role in several microbe-microbe interactions [3].

Microbial VOCs are composed by lipophilic chemical structures with high vapor pressure, which pass through biological membranes (live organism) to be released into the environment where the living organism is found [4]. Many of these compounds are by-products of primary metabolism yielded through different pathways, such as fermentation, aerobic heterotrophy, amino acid catabolism, sulfur reduction, and terpenoid biosynthesis. It is known about 2,000 mVOCs, with the most common belonging to the chemical classes of alcohols, alkanes, alkenes, aromatic compounds, nitrogen- and sulfurcontaining compounds, and terpenes [5].

When subjected to co-culture techniques, microorganisms can activate silenced genes, leading to the production mVOCs $[6,7]$ that will play important role in several microbe-microbe interactions, including antagonism. This latter relationship has been used in studies aiming to identify mVOCs responsible for performing inhibitory activity against phytopathogens [8,9]. In this context, rhizosphere microorganisms, especially bacterial strains, play an important role in agriculture, protecting plants against phytopathogens [10], and being used as biological control agents in the effective management of plant diseases [11]. It is noteworthy the importance of actinobacteria (filamentous Gram-positive bacteria), which are rich sources of secondary metabolites [12,13], being responsible for the production of various VOCs.

Actinobacteria have great potential of VOCs production, with the most frequently identified compounds being butan-1-ol, 2-methylpropan-1-ol, 3-methylbut-3-en-1-ol, 3-methylbutan-1-ol, dimethyl disulfide, dimethyl trisulfide, 2-phenylethanol and geosmin $[13,14]$. This latter mVOC is a sesquiterpene responsible for the "after-the-rain" smell. Streptomyces species are responsible for producing many VOCs, most of them derived from terpenoids with antifungal properties and biocontrol effectiveness. For example, methylvinylketone, produced by S. griseoruber, is reported to inhibit the germination of spores from the fungus Cladosporium cladosporioides [15]. Likewise, VOCs produced by S. alboflavus and S. philanthi promoted growth inhibition of Fusarium moniliforme, F. fujikuroi, Aspergillus flavus, A. ochreceus, A. niger, Penicillium citrinum, Rhizoctonia solani, Pyricularia grisea and Bipolaris oryzae [16]. Additionally, VOCs from S. globisporus inhibited the mycelial growth, spore germination and appressoria formation of Botrytis cinerea in tomato fruits and protected them against post-harvest gray mold caused by this fungus [17]. 

the compounds under relatively mild conditions. The extraction occurs in the absence of solvents and it is based on the partition equilibrium of analytes between the sample matrix and the extraction phase [18,19]. Different types of mixed fibers can be used as matrix, including polydimethylsiloxane-divinylbenzene-carboxene (PDMS/DVB/CAR), polydimethylsiloxanedivinylbenzene (PDMS/DVB) and polydimethylsiloxane-carboxene (PDMS/CAR). Therefore, an effective extraction of VOCs using HS-SPME requires the optimization of the protocol, varying important parameters, such as fiber coating and headspace conditions (e.g., extraction time and temperature) [20]. After HS-SPME procedure, the separation and identification of the extracted compounds is done through the hyphenated technique of gas chromatography-mass spectrometry (GC-MS) $[21,22]$. produced by twenty actinobacteria strains, isolated from rhizosphere associated to Caatinga growing plants, with antagonist effect against the phytopathogen fungus Colletotrichum musae. It is noteworthy that Caatinga is a semi-arid ecosystem found exclusively in Brazil. Because Amycolatopsis sp. (ACTB-290) and Streptomyces sp. (ACTB-77) were the most promising strains against Colletotrichum musae, VOCs produced by these microorganisms under axenic and co-culture conditions were investigated more deeply.

\section{Materials and methods}

\section{Microorganisms and culture medium}

Twenty strains of actinobacteria from the rhizosphere of Caatinga plants (Table 1) used in this work were obtained from the

Laboratory of Phytopathology at Embrapa Tropical Agro-business (CNPAT, Fortaleza, Ceará, Brazil).

Table 1. Actinobacteria strains isolated from Caatinga plants and their respective geographic locations.

\begin{tabular}{lcc}
\hline \multicolumn{1}{c}{ Strain code } & Plant Source & Geographic location \\
\hline ACTB-10 & Mimosa arenosa & \\
ACTB-25 & M. arenosa & $4^{\circ} 11^{\prime 2} 26,62^{\prime \prime} \mathrm{S} 38^{\circ} 29^{\prime} 50,78^{\prime \prime} \mathrm{O}$ \\
ACTB-32 & Bauhinia forficata & \\
\hline ACTB-77 & Combretum leprosum & $6^{\circ} 6^{\prime} 1.188^{\prime \prime} \mathrm{S} 39^{\circ} 23^{\prime} 0.29^{\prime \prime} \mathrm{O}$ \\
\hline ACTB-166 & Sideroxylon obtusifolium & $4^{\circ} 33^{\prime} 4.44^{\prime \prime} \mathrm{S} 39^{\circ} 37^{\prime} 53.18^{\prime \prime} \mathrm{O}$ \\
\hline ACTB-171 & Croton blanchetianus & $5^{\circ} 12^{\prime} 55.97^{\prime \prime} \mathrm{S} 37^{\circ} 00^{\prime} 57.07 " \mathrm{O}$ \\
\hline ACTB-172 & Myracrodruon urundeuva & \\
ACTB-177 & Cordia trichotoma & $4^{\circ} 33^{\prime} 4.44^{\prime \prime} \mathrm{S} 39^{\circ} 37^{\prime} 53.188^{\prime \prime} \mathrm{O}$ \\
ACTB-227 & C.trichotoma & \\
ACTB-242 & Cereus jamacaru & \\
\hline
\end{tabular}




\begin{tabular}{lc}
\hline ACTB-246 & C. jamacaru \\
ACTB0-282 & Commiphora leptophloeos \\
ACTB-285 & Anadenanthera colubrina \\
ACTB-286 & A. colubrina \\
ACTB-290 & A. colubrina \\
ACTB-291 & A. colubrina \\
ACTB-292 & A. colubrina \\
ACTB-296 & A. colubrina \\
ACTB-299 & Croton blanchetianus \\
\hline ACTB-305 & Licania rigida \\
\hline
\end{tabular}

The strain of the phytopathogenic fungus Colletotrichum musae (MMBF226/12) was donate by the Biologic Institute of São Paulo. C. brevisporum (CMM-1179), Lasiodiplodia brasiliense (strains CMM-2248 and CMM-2253), L. theobramae (CMM22004), L. hormozganensis (CMM-2211) and L. viticola (CMM-2252) strains were from Federal Rural University of Pernambuco (UFRPE), Collection of phytopathogenic fungi culture-Prof. Maria Menezes (CMM collection).

All microorganisms were cultured on commercially available potato dextrose agar medium (39.0 g.L $\left.{ }^{-1}\right)$ from $\mathrm{Kasvi}^{\circledR}(84.4 \%$ of potato broth, $8.4 \%$ of dextrose and $7.2 \%$ of bacteriological agar).

\section{Screening on actinobacteria producing VOCs with antifungal activity against $C$. musae}

The selection of VOCs-emitting actinobacteria strains with antifungal activity followed the methodology described in literature [23] and C. musae was used as target fungus. The 20 strains of actinobacteria were grown, separately, in PDA for 5 days. Subsequently, a $5 \mathrm{~mm}$ disk from each of these microorganisms was transferred to one side of a bi-Petri Himedia® plate (Figure 1S) and the plate was kept for 2 days under a static condition at $28^{\circ} \mathrm{C}$. After that, a $5 \mathrm{~mm}$ disk of mycelium of the $C$. musae, previously grown in PDA for 5 days, was inoculated on the other side of the bi-Petri plate (Figure 1S). For each experiment, control plates were prepared containing only the C. musae (absence of actinobacteria). All the plates were wrapped with double layers of Kasvi@ parafilm and incubated under static conditions at $28{ }^{\circ} \mathrm{C}$ for 5 days. The inhibition of mycelial growth of the fungus was expressed in percentage $(\%)$, considering the average diameters of the fungus growth in the control plate and in the experiment [24]. The diameters were measured with a digital caliper, model DANIU (100mm), from Vernier High Precision Carbon Fiber Compounds. Bioassays were performed in triplicates and the obtained data were analyzed according to one-way ANOVA and the values were considered significant when $p<0.05$ (GraphPad prism). 
The antifungal activity of VOCs emitted by actinobacteria Amycolatopsis sp. (ACTB-290) and Streptomyces sp. (ACTB-77) was assayed against the phytopathogenic fungus $C$. musae using Petri dish Kasvi ${ }^{\circledR}$ (80 mm diameter) [25]. A cell suspension $(10 \mu \mathrm{L})$ of the actinobacteria was inoculated in a Petri dish containing PDA culture medium. The plate was incubated for 48

$\mathrm{h}$, at $28{ }^{\circ} \mathrm{C}$, under static condition. After this period, a $5 \mathrm{~mm}$ disk of the fungus mycelium was inoculated in the center of another $80 \mathrm{~mm}$ diameter Petri dish containing PDA (Figure 2S). Then, the sides of the Petri dishes containing the microorganisms (actinobacteria and fungus) were overlapped, so that the distance between the inoculum was $1.5 \mathrm{~cm}$ (Figure 2S) and the actinobacteria culture was placed at the bottom [26]. The plates were wrapped with double layers of Kasvi ${ }^{\circledR}$ parafilm and incubated under static conditions at $28{ }^{\circ} \mathrm{C}$ for 5 days. As control experiment, Petri dishes were inoculated with the fungus exposed to PDA culture medium only. The diameter ( $\mathrm{mm}$ ) of the fungus mycelium was measured daily until completed fungus growing (at the fifth day) in the control experiment. Experiments were carried out in triplicate and all data obtained were analyzed according to one-way ANOVA, which determined a significant difference with $p<0.05$ using the computer program GraphPad prism.

\section{Antifungal activity of VOCs produced by ACTB-290 strain against other phytopathogen fungi}

The antifungal activity of VOCs emitted by actinobacteria Amycolatopsis sp. (ACTB-290) was assayed against the phytopathogenic fungal strains $C$. brevisporum, Lasiodiplodia brasiliense, L. theobramae, L. hormozganensis, L. brasiliense and L. viticola, following the same procedure aforedescribed for C. musae. In this case, the diameter (mm) of each mycelium was measured daily until completed fungus growing in the control experiment, which varied as follows: C. brevisporum (8 days), L. theobramae (8 days), L. brasiliense (strain CMM-2248: 5 days; strain CMM-2253: 4 days), L. hormozganensis (4 days) and L. viticola (3 days).

\section{Molecular identification of actinobacteria strains ACTB-290 and ACTB-77}

The genomic DNA of all strains was extracted using the Bacterial Genomic DNA purification kit (HIMEDIA), following the manufacturer's instructions. DNA quantification was performed by the NanoDrop® 2000c spectrophotometer (Thermo Fisher Scientific), version 1.0, and the concentration of $10 \mathrm{ng} \mu \mathrm{L}^{-1}$ was then diluted and stored at $-20{ }^{\circ} \mathrm{C}$. The nucleotide sequence of the $16 \mathrm{~S}$ genomic region of ribosomal DNA (rDNA) was amplified by polymerase chain reaction (PCR) using primers $27 \mathrm{~F}\left(5^{\prime}-\right.$ GAGTTTGATCMTGGCTCAG-3 ') and 1492R (5'-ACGGYTACCTTGTTACGACTT-3'). The PCR mixtures (50 $\mu$ L) 
contained $6.25 \mu \mathrm{L}$ of genomic DNA $\left(10 \mathrm{ng} \mu \mathrm{L}^{-1}\right), 10 \mu \mathrm{L}$ of $5 \mathrm{x}$ buffer, $1 \mu \mathrm{L}$ of dNTP $(10 \mathrm{mM}), 2 \mu \mathrm{L}$ of $\mathrm{MgCl}_{2}(25 \mathrm{mM}), 0.8$ $\mu \mathrm{L}$ of each primer $(10 \mathrm{mM}), 0.5 \mu \mathrm{L}$ of GoTaq polymerase $\left(5 \mathrm{U} \mathrm{LL}^{-1}\right)$ and $28.65 \mu \mathrm{L}$ of sterile ultrapure water.

Flexigene ${ }^{\circledR}$ thermal cycler program was used in the PCR amplifications and it included an initial denaturation step at $94{ }^{\circ} \mathrm{C}$ for $2 \mathrm{~min}$, followed by 35 denaturation cycles at $94{ }^{\circ} \mathrm{C}$ for $60 \mathrm{~s}$, annealing at $56{ }^{\circ} \mathrm{C}$ for $45 \mathrm{~s}$ and extension $72{ }^{\circ} \mathrm{C}$ for $60 \mathrm{~s}$, with a final extension at $72{ }^{\circ} \mathrm{C}$ for $10 \min$ [27]. The PCR products were separated by electrophoresis on $1.5 \%$ agarose gel in $1 \mathrm{X}$ Tris Borate EDTA buffer, stained with ethidium bromide $\left(0.5 \mathrm{mg} \mathrm{mL}^{-1}\right)$ for 1 min and visualized under $\mathrm{UV}$ transluminator. After checking the amplified bands, $40 \mu \mathrm{L}$ aliquots of each PCR product were purified and sequenced by Macrogen Inc., South Korea (http://www.macrogen.com).

The nucleotide sequences were edited using the BioEdit program version 7.0.5 [28] and were subjected to identity verification using the basic local alignment search tool (Basic Local AlignmentSearch Tool = BLASTn) from GenBank, followed by manually alignment using ClustalW with strings previously published and deposited in GenBank (NCBI, http://www.ncbi.nlm.nih.gov). The phylogenetic analyzes of maximum parsimony were performed using the PAUP 4.0 program beta 10. For the analysis of maximum parsimony, the following options were selected: heuristic searches, treebisection-reconnection (TBR), branch swapping and MULTREES. The statistical support of the tree was tested using bootstrap analysis with 1,000 replicates.

\section{Scanning Electron Microscope (SEM) imaging of $C$. musae hyphae}

Morphological analyses of C. musae was performed in a scanning electron microscope (SEM, Zeiss DSM, model $940 \mathrm{~A}$ ), at an acceleration voltage of $15 \mathrm{kV}$. Samples (1-5 mm cubes) of PDA containing the fungus hyphae were submerged in a $2 \mathrm{~mL}$ Eppendorf vial with Karnovsky solution in a proportion of 10 times the volume of samples in the vial. After $1 \mathrm{~h}$ at room temperature under static condition, Karnovsky solution was removed, and samples were washed during $10 \mathrm{~min}$ with $0.1 \mathrm{M}$ phosphate buffer solution $(3 \times 2 \mathrm{~mL})$. An aliquot (enough to cover the samples) of $1 \%$ solution of $\mathrm{OsO}_{4}$ was added into the Eppendorf vial and maintained for $1 \mathrm{~h}$ at room temperature under static condition. After removing the solution, the samples were washed three times (15 min between each washing) with distilled water, followed by dehydration with increasing concentration of ethanol solution (20,40, 60, 80 and $100 \%)$. Subsequently, the samples were subjected to the critical drying point in the Quorum critical point drying apparatus model K850 and placed in metallic sample holders (stabs). After being coated with a gold layer, the samples were analyzed in SEM equipment. 
The experiment to optimize mVOCs extraction by HS-SPME was carried out with the actinobacteria Amycolatopsis sp. (ACTB-290) and it was based on a similar experiment described in the literature [29]. The following solid phase microextraction fibers (SPME) from Supelco ${ }^{\circledR}$ (needle size: $24 \mathrm{ga}$; fiber L: $1 \mathrm{~cm}$ ) were used: polydimethylsiloxanedivinylbenzene-carboxene (PDMS/DVB/CAR; $\left.\mathrm{d}_{\mathrm{f}}: 50 / 30 \mu \mathrm{m}\right)$, polydimethylsiloxane-divinylbenzene (PDMS/DVB; $\left.\mathrm{d}_{\mathrm{f}}: 65 \mu \mathrm{m}\right)$ and polydimethylsilzane-carboxene (PDMS/CAR; $\left.\mathrm{d}_{\mathrm{f}}: 85 \mu \mathrm{m}\right)$. The fibers were conditioned prior to use following the manufacturer's recommendations.

The actinobacterial strain was inoculated in Petri dishes containing PDA medium and incubated for 7 days at $28{ }^{\circ} \mathrm{C}$ under static condition. Subsequently, $10 \mathrm{~mL}$ of the still liquid PDA medium was added to $20 \mathrm{~mL}$ EPA Sigma Aldrich ${ }^{\circledR}$ vial (PTFE/silicone septum). After solidification of the medium in the vial, a $5 \mathrm{~mm}$ disk of the previously inoculated actinobacteria was added. The vial containing the inoculum was immediately closed with silicone septum, capped with thread, and maintained 5 days at $28{ }^{\circ} \mathrm{C}$ under static condition. Then, the vial was placed in a water bath (at 30 or $50{ }^{\circ} \mathrm{C}$ ) and, after 5 min, the fiber was inserted through a hole in the septum $(1 \mathrm{~cm}$ above the microorganism), Figure $3 \mathrm{~S}$. The time of VOCs extraction varied from 10 to $40 \mathrm{~min}$. After each extraction time, the fiber was removed from the vial and it was inserted into the gas chromatograph (GC-FID) injector (Shimadzu model QP-2010s) for 5 min at $250{ }^{\circ} \mathrm{C}$ (splitless mode) for thermal desorption of the analytes. GC-FID was equipped with a DB-5MS capillary column (30 m x $0.25 \mathrm{~mm}$ x $0.25 \mu \mathrm{m}$, film thickness) from Agilent J\&W GC Columns (Santa Clara, CA, USA). Analyzes conditions were: GC oven temperature $40{ }^{\circ} \mathrm{C}$ for 2 min; 10 ${ }^{\circ} \mathrm{C} / \mathrm{min}$ up to $195{ }^{\circ} \mathrm{C} ; 7^{\circ} \mathrm{C} / \mathrm{min}$. up to $220{ }^{\circ} \mathrm{C} ; 10{ }^{\circ} \mathrm{C} / \mathrm{min}$ up to $260{ }^{\circ} \mathrm{C}$. Volumetric flow of the mobile phase (helium gas) of $0.59 \mathrm{~mL} / \mathrm{min}$; detector temperature was $250^{\circ} \mathrm{C}$.

All experiments were carried out in triplicate, resulting 72 analyzes. Number of peaks and areas were presented as total mean values of all compounds. The obtained data were analyzed using the free software for statistical computing R Program (The R foundation for statistical computing; Vienna, Austria, 2014).

HS-SPME and GC-MS analysis of VOCs produced by Amycolatopsis sp. (ACTB-290) and Streptomyces sp. (ACTB-77) under axenic culture and co-culture with C. musae

mVOCs Extraction by HS-SPME

Experiments of VOCs extraction were performed with the actinobacteria Amycolatopsis sp. (ACTB-290) and Streptomyces sp. (ACTB-77) under axenic culture and co-cultured with C. musae. All microorganisms were previously grown, separately, 
in Petri dishes containing PDA medium for 5 days at $28^{\circ} \mathrm{C}$ under static condition. Microbial VOCs extraction by HS-SPME was carried out using the optimized conditions (fiber: PDMS/DVB/CAR; extraction time: 30 min; extraction temperature: 50 ${ }^{\circ} \mathrm{C}$ ).

Three 20 mL EPA Sigma Aldrich ${ }^{\circledR}$ vials (PTFE/silicone septum) were used, two for axenic cultures (actinobacteria and fungus) and one for co-culture (actinobacteria together with fungus). PDA medium (10 mL) was added to each vial of axenic culture and the microorganisms (actinobacteria and fungus) were inoculated in the center of each vial. In the case of the coculture experiment, $3 \mathrm{~mL}$ of the culture medium was added to one side of the vial in a horizontal position. After solidification of the PDA medium, an additional $3 \mathrm{~mL}$ of the medium was added on the opposite side of the same vial (Figure 4S). Initially, a $5 \mathrm{~mm}$ disk of the actinobacteria strain previously inoculated in the Petri dish was added to the vial corresponding to its individual culture and another $5 \mathrm{~mm}$ disk to one side of the vial of the co-culture experiment. After $48 \mathrm{~h}$, a $5 \mathrm{~mm}$ disk of the fungus previously inoculated in the Petri dish was added to the flask corresponding to the individual culture experiment and another $5 \mathrm{~mm}$ disk to the other side of the flask of the co-culture experiment (opposite side of the actinobacteria strain).

After 5 days of microorganisms' inoculation, all vials were placed in water bath at $30{ }^{\circ} \mathrm{C}$ and, 5 min later, the PDMS/DVB/CAR fiber was inserted through a hole in the septum (1 cm above the microorganism). Microbial VOCs were extracted for $30 \mathrm{~min}$ at $30{ }^{\circ} \mathrm{C}$. Then, the fiber was removed and inserted into the GC-MS injector using the same conditions previously described in the experimental of optimization of mVOCs extraction by HS-SPME. The experiments were carried out in triplicate and PDA medium (without microorganism) was used as control, resulting in 18 analyses (3 axenic culture of ACTB-290; 3 axenic culture of ACTB-77; 3 axenic culture of $C$. musae; 3 co-culture of ACTB-290 + C. musae; 3 co-culture of ACTB-77 + C. musae; 3 control).

mVOCs Analysis by GC-MS

Extracted mVOCs were analyzed in a gas chromatographer (GC model 7890B) coupled to a mass spectrometer (MS model 5977A MSD) from Agilent Technologies Spain. Compounds were separated in GC using the same conditions previously described in the experimental of optimization of mVOCs extraction by HS-SPME. Electron impact (70 eV) MS data were recorded with $\mathrm{m} / \mathrm{z}$ from 50 to 500 Daltons with intervals of $0.5 \mathrm{~s}$.

Compound`s identification was done by comparison of the mass spectra obtained for each compound with the one reported in mass spectral libraries [30], including NIST 05 and NIST 27 (National Institute of Standards and Technology, Gaithersburg, USA). Additionally, Kovats index was calculated for each compound using a mixture of saturated n-alkanes C7-C30 (SigmaAldrich $\left.^{\circledR}\right)$, followed by comparison with literature data $[30,31,32]$. 


\section{Statistical analysis of the experiments}

From the data obtained, three matrices were elaborated where the rows corresponded to the samples of $C$. musae, ACTB-77 and the respective co-cultures of $C$. musae with ACTB-77 (Matrix 1) and the columns contained the peak values obtained regarding mVOCs. Matrix 2 was elaborated in a similar way to Matrix 1 but containing the peak values for the samples of $C$. musae, ACTB-290 and co-culture of C. musae and ACTB-290. Finally, Matrix 3 was prepared including all rows and columns of Matrices 1 and 2. With these data sets organized, the matrices were autoscaled and then subjected to a principal component analysis (PCA) to observe differences and similarities between the samples studied. For matrix 1 and 2 , two principal components (PC) were used and for matrix 3, three PCs were used. PCA calculations were performed in PLS-ToolBox 5.2 and Matlab ${ }^{\circledR} 2010$ a was used.

\section{Results and Discussion}

\section{Screening on actinobacteria producing VOCs with antifungal activity against $C$. musae}

Twenty strains of actinobacteria isolated from rhizosphere of plants from Caatinga biome (Table 1) were assayed for their ability to produce VOCs capable to inhibit the growth of $C$. musae, a phytopathogen fungus responsible for causing anthracnosis in banana culture [33]. Experiments were performed in bi-Petri dishes (Figure 1) and growth inhibition (GI) was recorded after 5 days of culturing. Figure 1 shows that all actinobacteria strains assayed promoted fungal growth inhibition (GI 50.3-73.7\%) and, among them, ACTB-77 (GI 68.0\%) and ACTB-290 (GI 73.7\%) promoted the highest inhibitory effect. Therefore, these two strains were selected for molecular identification and further VOCs investigation under axenic and coculture conditions. 


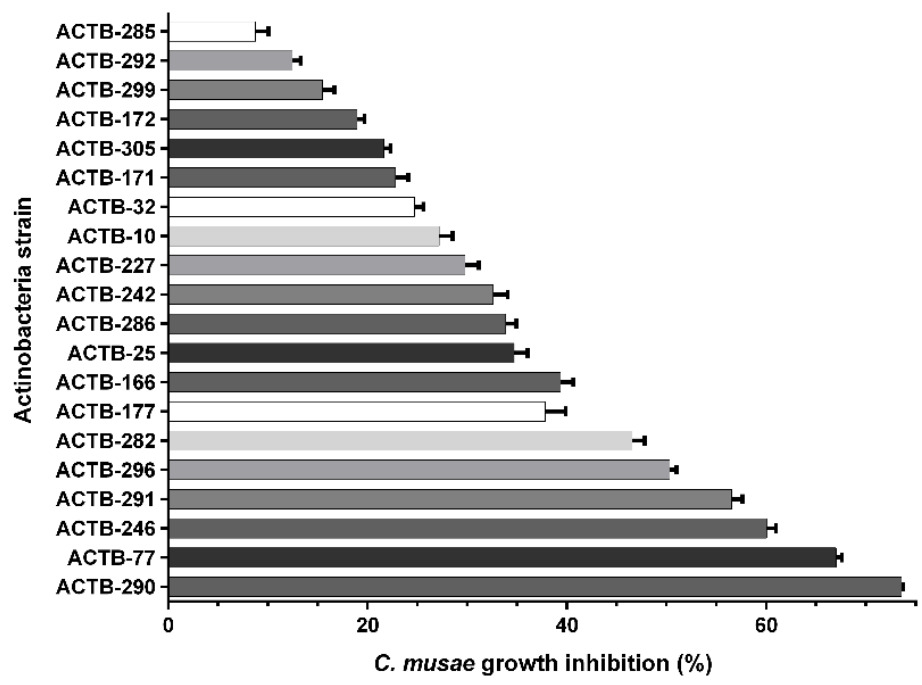

280 Figure 1. Growth inhibition (\%) of $C$. musae by volatile organic compounds (VOCs) produced by the twenty actinobacteria strains assayed.

Molecular identification of strains ACTB-77 and ACTB-290

The promising antifungal potential of VOCs produced by strains ACTB-77 and ACTB-290 in the growth inhibition of $C$. musae motivated their identification by molecular approach. Strains ACTB-77 and ACTB-290 showed 99\% similarity to Streptomyces spp. and Amycolatopsis spp., respectively According to the most parsimonious phylogenetic tree (Figure 2) obtained from the 16S sequence data set of strains of the genus Amycolatopsis, the sequence of strain ACTB-290 was grouped in a clade with strains of Amycolatopsis sp. with 100\% bootstrap support, strain while ACTB-77 was grouped in a distinct clade of the genus Streptomyces sp. 


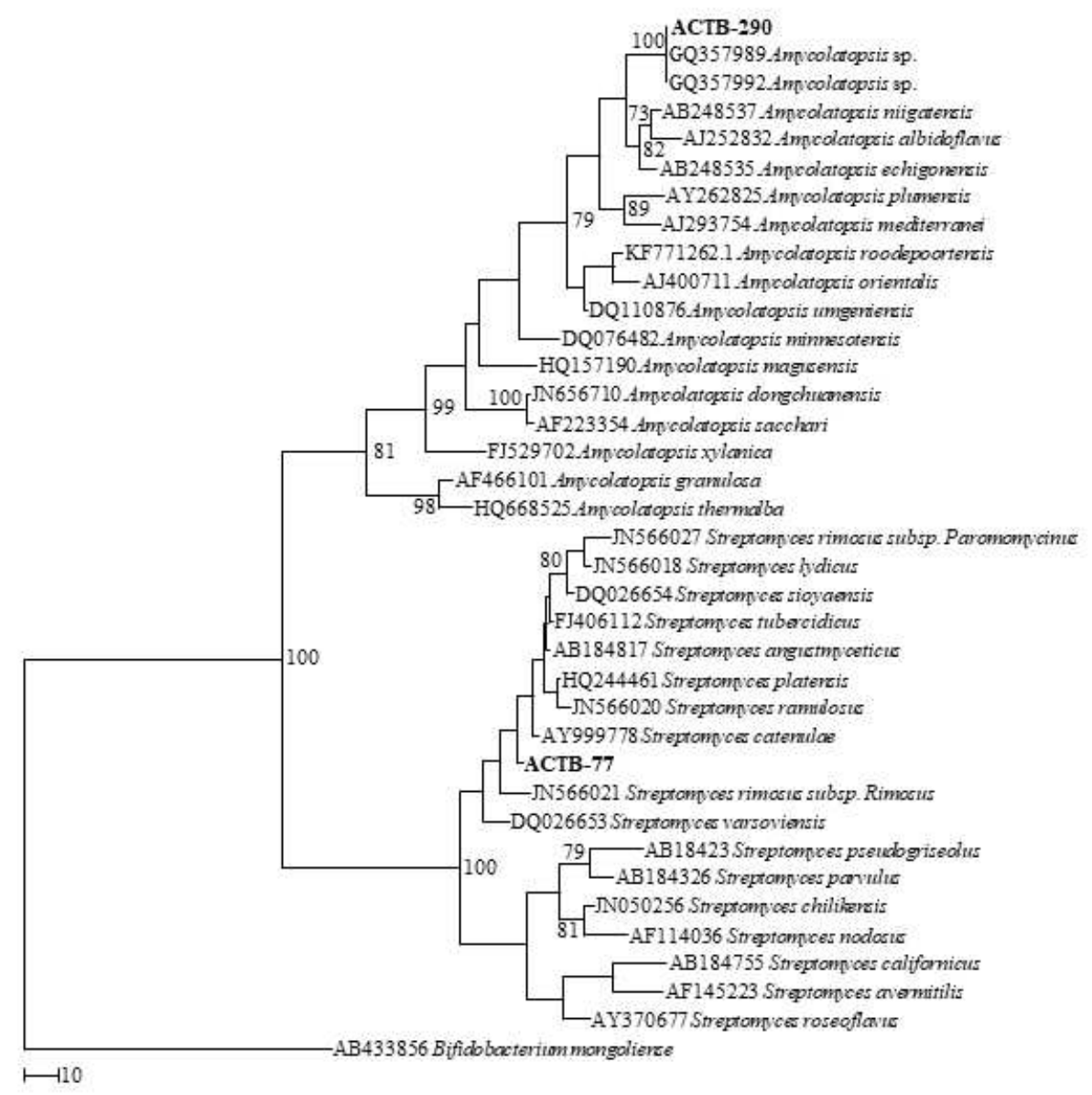

291 Figure 2. Phylogenetic tree inferred by maximum parsimony (MP) of data from the $16 \mathrm{~S}$ genomic region of the rDNA for sequences of the genera Amycolatopsis and Streptomyces. Bootstrap values (> 70\%) with 1000 repetitions are shown in the respective branch. Bifidobacterium mongoliense was used as an external group. The sequences in this study are highlighted in bold.

To evaluate the antifungal activity of VOCs produced by the two selected strains in more detail, a new experiment was carried out to monitor the growth inhibition starting from the first day of microbial inoculation until the fifth day of experiment when the control fungus strain occupied 100\% of the Petri dish. As observed in Figure 3, the two strains promoted similar growth inhibitions of the fungus, which initiated from the second day of incubation and continued until the end of the experiment. 


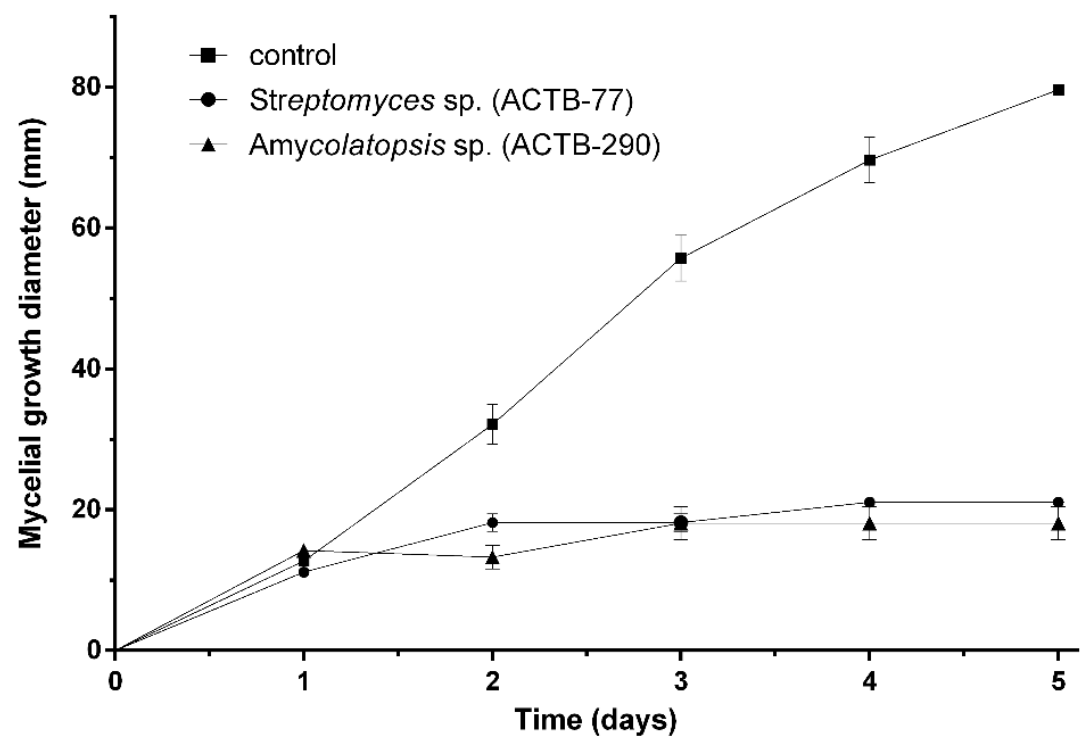

Figure 3. Results from the antifungal activity of Streptomyces sp. (ACTB-77) and Amycolatopsis sp. (ACTB-290) against $C$. musae.

\section{SEM imaging of C. musae hyphae}

The Petri dishes from the fifth day of the aforementioned assays (control and co-culture) were used to investigate the mVOCs
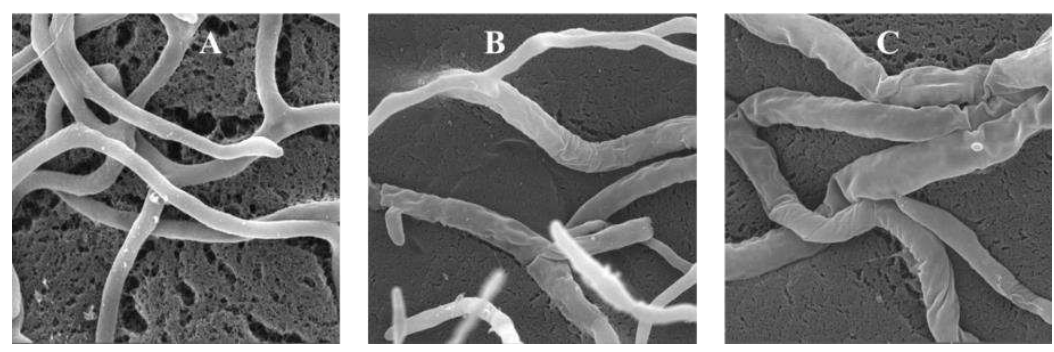

Figure 4. SEM imaging $(20 \mu \mathrm{M})$ of $C$. musae hyphae in the fifth day of experiment: (A) under axenic culture (control); (B) in co-culture with Streptomyces sp.; (C) in co-culture with Amycolatopsis sp.

Figure 4A shows the perfect stage of $C$. musae hyphae under axenic cultured (control), with fungal cell structure having straight, cylindrical and long hyaline (non-septate hyphae), and their walls remaining smooth and shiny [34]. The effect of VOCs from actinobacteria ACTB-77 strain on C. musae hyphae is observed in Figure 4B. Although most of the hyphae showed wrinkled and withered aspects, a small portion of cell filaments was partially unharmed, and no breakage of the hyphae was observed. SEM image of fungus hyphae from co-culture experiment with ACTB-290 strain is depicted in Figure 4C. In 
this case, it was observed the deformation of all filaments of residual cells, with wrinkled, withered (loss of turgor) and brittle aspects, suggesting a possible leakage of the intracellular material.

Thus, SEM imaging analysis of $C$. musae hyphae revealed that VOCs produced by the actinobacteria strains during coculture experiments promote deformation of the fungal cell filaments. The highest antifungal activity of VOCs from Amycolatopsis sp. (ACTB-290) was microscopically proven through the greatest damage observed in the fungal hyphae.

The antifungal potential of VOCs from Amycolatopsis sp. against $C$. musae motivated the investigation of the activity of this strain against other species of phytopathogenic fungi, one from Colletotrichum genus (C. brevisporum) and five species of was repeated using the same conditions afore discussed, and the results are presented in Figure 5. brevisporum (GI 76.0\%), L. theobramae (GI 68.0\%) and L. harmozganensis (GI 54.4\%). Comparison of these results with species. 

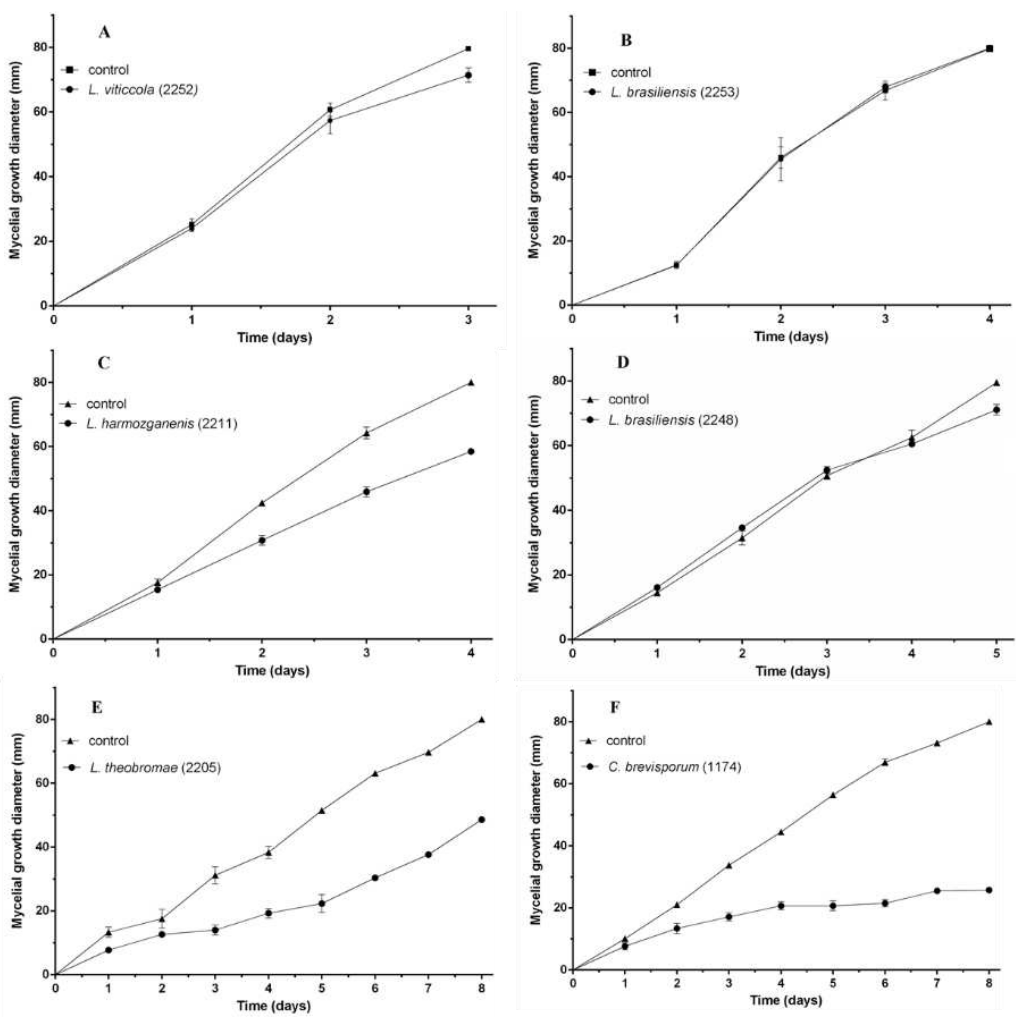

Figure 5. Results from the antifungal activity of Amycolatopsis sp. (ACTB-290) against: (A) Lasiodiplodia viticola; (B) L. brasiliensis-2253; (C) L. brasiliensis-2248; (D) L. theobromae; (E) Colletotrichum brevisporum. Fungus strain under axenic culture is the control experiment.

Identification of VOCs produced by Amycolatopsis sp. (ACTB-290) and Streptomyces sp. (ACTB-77) under axenic culture and co-culture with $C$. musae

Optimization of the VOCs extraction from Amycolatopsis sp. (ACTB-290) by HS-SPME

As already mentioned, studies on mVOCs first require the experiment optimization by varying fiber coating and headspace conditions [20]. Thus, experiment optimization involved the actinobacteria ACTB-290 by varying fiber coating (PDMS/DVB/CAR, PDMS/DVB and PDMS/CAR), the extraction time (10, 20, 30 and $40 \mathrm{~min})$ and temperature (30 and 50 ${ }^{\circ} \mathrm{C}$ ). Microbial VOCs were analyzed by GC-MS and evaluated in relation to the quantity and area of the peaks in each experiment. Based on the variation of the observed error, it was concluded that temperature is a significant factor, being more important than the extraction time. However, when analyzing the interactions between fiber, extraction time and temperature, it was noticed that, for this matrix, these variables are independent. After all data analyses of the optimization experiments of 
PDMS/DVB/CAR, extraction time $30 \mathrm{~min}$ and extraction temperature $50{ }^{\circ} \mathrm{C}$.

The actinobacteria strains, Streptomyces sp. (ACTB-77) and Amycolatopsis sp. (ACTB-290), and the phytopathogen fungus

C. musae were cultured (axenic and co-culture conditions) in PDA medium for mVOCs production. For all microorganisms,

HS-SPME experiments were performed using the optimized conditions (PDMS/DVB/CAR, $50^{\circ} \mathrm{C}$ and $30 \mathrm{~min}$ ). Analyses of

their respective VOCs were done by GC-MS and their composition are displayed at Table $\mathbf{2}$.

Table 2. Volatile organic compounds (VOCs) produced by the rhizosphere actinobacteria Streptomyces sp. (ACTB-77) and Amycolatopsis sp. (ACTB-290) strains, and the phytopathogen fungus Colletotrichum musae (CM) under axenic and coculture conditions.

\begin{tabular}{|c|c|c|c|c|c|c|c|}
\hline \multirow{2}{*}{\multicolumn{3}{|c|}{ Compound }} & \multirow{2}{*}{\multicolumn{3}{|c|}{$\begin{array}{l}\text { Axenic culture } \\
\text { Peak area }(\%)\end{array}$}} & \multirow{2}{*}{\multicolumn{2}{|c|}{$\frac{\text { Co-culture }}{\text { Peak area }(\%)}$}} \\
\hline & & & & & & & \\
\hline $\mathrm{Nr}$. & Name & RI & $\mathrm{CM}$ & ACTB-77 & ACTB-290 & $\begin{array}{c}\text { CM+ } \\
\text { ACTB-77 }\end{array}$ & $\begin{array}{c}\text { CM+ } \\
\text { ACTB-290 }\end{array}$ \\
\hline 1 & 3-Methyl-butan-1-ol & 731 & $23.82 \pm 2.07$ & $1.77 \pm 0.11$ & - & $6.42 \pm 0.75$ & $5.76 \pm 0.09$ \\
\hline 2 & 2-Methyl-butan-1-ol & 732 & $5.85 \pm 0.31$ & $0.98 \pm 0.08$ & - & $2.43 \pm 0.26$ & $2.51 \pm 0.02$ \\
\hline 3 & 3-Methyl-pentan-2-one & 747 & - & $2.03 \pm 0.04$ & - & - & - \\
\hline 4 & Dimethyl disulfide & 752 & - & - & $48.49 \pm 3.48$ & - & $2.18 \pm 0.68$ \\
\hline 5 & $\begin{array}{l}\text { 3-Methylbutan-1-ol } \\
\text { acetate }\end{array}$ & 869 & $0.96 \pm 0.04$ & - & - & - & - \\
\hline 6 & 2,4-Dithiapentane & 893 & & & $4.32 \pm 0.85$ & & $0.58 \pm 0.11$ \\
\hline 7 & 6-Methyl-heptan-2-one & 961 & - & - & $2.47 \pm 0.07$ & - & $0.77 \pm 0.06$ \\
\hline 8 & Dimethyl trisulfide & 976 & & & $21.14 \pm 3.50$ & & $0.76 \pm 0.25$ \\
\hline 9 & $\beta$-Myrcene & 980 & $1.14 \pm 0.22$ & $4.95 \pm 0.65$ & & $1.33 \pm 0.02$ & $0.66 \pm 0.07$ \\
\hline 10 & $(S)$-Verbenone & 985 & & & $0.94 \pm 0.04$ & & $0.36 \pm 0.05$ \\
\hline 11 & 2-Methylenebornane & 990 & & & $1.13 \pm 0.04$ & & $0.78 \pm 0.20$ \\
\hline 12 & $\alpha$-Phellandrene & 994 & $8.09 \pm 1.72$ & - & - & $11.94 \pm 0.39$ & $11.91 \pm 0.65$ \\
\hline 13 & $\alpha$-Terpinene & 1008 & $3.43 \pm 0.74$ & - & - & $4.22 \pm 0.05$ & $6.02 \pm 0.21$ \\
\hline 14 & $p$-Cymene & 1017 & $3.16 \pm 0.47$ & - & - & $3.50 \pm 0.11$ & $3.47 \pm 0.28$ \\
\hline 15 & $\beta$-Phellandrene & 1023 & $28.38 \pm 4.89$ & - & - & $43.01 \pm 1.53$ & $44.71 \pm 2.61$ \\
\hline 16 & 2-Methyl-2-bornene & 1023 & & & $2.42 \pm 0.26$ & & \\
\hline 17 & trans- $\beta$-Ocimene & 1042 & & $2.41 \pm 0.35$ & - & - & - \\
\hline 18 & Methyl 2-ethylhexanoate & 1048 & & & $2.49 \pm 0.04$ & & $0.53 \pm 0.01$ \\
\hline 19 & Not identified & 1058 & & & $2.13 \pm 0.74$ & & $1.29 \pm 0.54$ \\
\hline 20 & 1-Octanol & 1064 & & $1.52 \pm 0.24$ & & & \\
\hline 21 & Linalool & 1092 & & $38.53 \pm 5.19$ & & $6.08 \pm 0.49$ & \\
\hline 22 & 2-Nonanone & 1094 & & & $1.14 \pm 0.24$ & & $2.40 \pm 0.10$ \\
\hline 23 & 2-Nonanol & 1102 & & & & & $0.47 \pm 0.04$ \\
\hline 24 & Phenylethyl alcohol & 1106 & $7.44 \pm 0.62$ & & & $1.97 \pm 0.32$ & $3.31 \pm 0.30$ \\
\hline 25 & allo-Ocimene & 1123 & & $0.71 \pm 0.11$ & & & \\
\hline 26 & Not identified & 1129 & & $1.7 \pm 0.23$ & & & \\
\hline 27 & $\begin{array}{l}\text { Methyl(methylthio)meth } \\
\text { yl disulfide }\end{array}$ & 1135 & & & $1.95 \pm 0.07$ & & \\
\hline 28 & $p$-Menthone & 1148 & $0.97 \pm 0.26$ & & & $1.26 \pm 0.10$ & $1.93 \pm 0.06$ \\
\hline 29 & $p$-Mentha-1,5-dien-8-ol & 1160 & $0.72 \pm 0.06$ & & & $0.43 \pm 0.08$ & \\
\hline
\end{tabular}




\begin{tabular}{|c|c|c|c|c|c|c|c|}
\hline 30 & 2-Decanone & 1162 & & & $0.80 \pm 0.07$ & & $0.92 \pm 0.12$ \\
\hline 31 & Menthol & 1166 & $1.39 \pm 0.15$ & & & $0.31 \pm 0.03$ & $0.58 \pm 0.11$ \\
\hline 32 & Not identified & 1182 & & & $0.76 \pm 0.09$ & & \\
\hline 33 & $\begin{array}{l}p \text {-Mentha-1(7),2-dien-8- } \\
\text { ol }\end{array}$ & 1184 & $1.64 \pm 0.15$ & & & & $0.62 \pm 0.15$ \\
\hline 34 & $\alpha$-Terpineol & 1186 & & $1.10 \pm 0.63$ & & $1.68 \pm 0.08$ & \\
\hline 35 & 2-Methylisoborneol & 1191 & & & $2.27 \pm 0.05$ & & $1.51 \pm 0.24$ \\
\hline 36 & Thymol methyl ether & 1224 & & $5.06 \pm 0.55$ & & $0.95 \pm 0.05$ & \\
\hline 37 & Carvacrol methyl ether & 1232 & & $4.24 \pm 0.68$ & & $0.64 \pm 0.03$ & \\
\hline 38 & Geraniol & 1249 & & $1.86 \pm 0.43$ & & $0.49 \pm 0.03$ & \\
\hline 39 & Not identified & 1254 & & $1.55 \pm 0.25$ & & $0.80 \pm 0.09$ & \\
\hline 40 & Not identified & 1261 & & & $1.64 \pm 0.17$ & & $0.42 \pm 0.09$ \\
\hline 41 & 1-Decanol & 1264 & & $0.79 \pm 0.07$ & & & \\
\hline 42 & Not identified & 1284 & & $0.75 \pm 0.04$ & & $0.23 \pm 0.01$ & \\
\hline 43 & 2-Undecanone & 1296 & & & $0.93 \pm 0.11$ & & $0.35 \pm 0.05$ \\
\hline 44 & 2-Undecanol & 1303 & & & & & $0.65 \pm 0.05$ \\
\hline 45 & Not identified & 1330 & & $0.52 \pm 0.05$ & & & \\
\hline 46 & Silphinene & 1335 & $0.42 \pm 0.06$ & & & $1.33 \pm 0.49$ & $0.43 \pm 0.02$ \\
\hline 47 & 2-Undecenal & 1337 & & $0.71 \pm 0.10$ & & & \\
\hline 48 & 2-Dodecanone & 1362 & & & $1.45 \pm 0.05$ & & $0.19 \pm 0.05$ \\
\hline 49 & Not identified & 1370 & & & $0.87 \pm 0.04$ & & $0.48 \pm 0.05$ \\
\hline 50 & 2-Dodecanol & 1375 & & & & & $0.28 \pm 0.03$ \\
\hline 51 & Not identified & 1401 & & $1.69 \pm 1.19$ & & $0.66 \pm 0.05$ & \\
\hline 52 & Geosmin & 1410 & & $17.25 \pm 2.55$ & & $3.08 \pm 0.16$ & \\
\hline 53 & Not identified & 1446 & & & & & $0.30 \pm 0.04$ \\
\hline 54 & 2-Tridecanone & 1464 & & & $0.67 \pm 0.04$ & & $0.31 \pm 0.07$ \\
\hline 55 & Not identified & 1470 & & $1.03 \pm 0.14$ & & & \\
\hline 56 & Not identified & 1470 & & & & & $0.39 \pm 0.03$ \\
\hline 57 & $\beta$-Chamigrene & 1476 & $0.91 \pm 0.19$ & & & $0.48 \pm 0.05$ & $0.39 \pm 0.05$ \\
\hline 58 & $\gamma$-Gurjunene & 1479 & & $0.39 \pm 0.08$ & & & \\
\hline 59 & Aristolochene & 1486 & $7.56 \pm 1.41$ & & & $3.50 \pm 0.19$ & \\
\hline 60 & $\alpha$-Selinene & 1498 & $3.60 \pm 0.75$ & & & $1.76 \pm 0.21$ & $1.54 \pm 0.20$ \\
\hline 61 & Viridiflorene & 1501 & & $0.39 \pm 0.06$ & & & \\
\hline 62 & Not identified & 1515 & & $0.73 \pm 0.13$ & & & \\
\hline 63 & 7-epi- $\alpha$-Selinene & 1522 & $0.53 \pm 0.11$ & & & $0.47 \pm 0.05$ & $0.20 \pm 0.02$ \\
\hline 64 & Selina-3,7(11)-diene & 1539 & & $6.92 \pm 0.09$ & & $1.43 \pm 0.12$ & \\
\hline 65 & 2-Tetradecanone & 1565 & & & $1.02 \pm 0.03$ & & \\
\hline 66 & $\begin{array}{l}\text { (Z)-8-Dodecen-1-ol } \\
\text { acetate }\end{array}$ & 1588 & & & $0.13 \pm 0.02$ & & \\
\hline 67 & 1-Tetradecanol & 1643 & & & $0.83 \pm 0.07$ & & \\
\hline 68 & $\alpha$-Eudesmol & 1647 & & $0.26 \pm 0.02$ & & & \\
\hline 69 & Mint sulfide & 1738 & & $0.2 \pm 0.02$ & & & \\
\hline
\end{tabular}

mVOCs profile under axenic culture

The study on VOCs produced by C. musae under axenic culture resulted in 18 recorded peaks, all of them identified by GC/MS 
$7.44 \pm 0.62 \%)$ and aristolochene $(\mathbf{5 9} ; 7.56 \pm 1.41 \%)$ represent $c a .75 \%$ of the mVOCs composition. Constituents from C. musae were distributed into four different cases, alcohols ( 3 compounds; $c a .37 \%$ total area), ester ( 1 compound; $c a .1 \%$ total area); monoterpenes ( 9 compounds; $c a .49 \%$ total area); sesquiterpenes ( 5 compounds; $c a .13 \%$ total area). It is noteworthy that no reports on VOCs produced by either C. musae or any other congener species were found in the literature for comparison purpose. Instead, the literature reports studies on VOCs either from bacteria strains with inhibition of Colletotrichum species $[35,36]$ and from plants/fruits contaminated with some fungus strains $[37,38]$.

Actinobacteria Streptomyces sp. (ACTB-77)

The study on VOCs produced by ACTB-77 strain under axenic culture yielded 27 peaks recorded in the GC chromatogram (Table 2). Among them, $74 \%$ of the compounds (20 peaks) were identified, together representing $c a .92 \%$ of the total area of all recorded peaks. The monoterpene linalool $(\mathbf{2 1} ; 38.53 \pm 5.19 \%)$ and the sesquiterpene geosmin $(\mathbf{5 2} ; 17.25 \pm 2.55 \%)$ were found as the main constituents, these two compounds accounting for $c a .56 \%$ of the mVOCs composition. The identified compounds belong to five different groups, alcohol ( 4 compounds; $c a .5 \%$ total area), ketone ( 1 compound; $c a .2 \%$ total area), aldehyde ( 1 compound; $c a .1 \%$ total area), monoterpene ( 9 compounds; $c a .76 \%$ total area) and sesquiterpene (5 compounds; ca. $8 \%$ total area). It is worth noting that sesquiterpene mint sulfide $(0.2 \pm 0.02 \%)$ is the only sulfur-containing compound produced by ACTB-77.

Some of these compounds, such as the alcohols, ketones and aldehydes, are reported as by-products from primary metabolic pathways of actinomycetes [5]. Previous investigations of VOCs profiling of Streptomyces isolates were reported in literature [14,26]. A total of twenty-six Streptomyces strains were assayed for their ability to produce VOCs [14]. In this study, 53 compounds were identified out of the 120 detected peaks. The compounds were classified as alkanes, alkenes, alcohols, esters, ketones, sulfur-compounds, and terpenes. As for ACTB-77, 3-methyl-butan-1-ol (1), 2-methyl-1-butanol (2), and geosmin (52) are among the most frequent compounds produced by the microorganisms. A similar study involved VOCs production of twelve Streptomyces strains, eleven of them from rhizosphere. Besides the presence of alcohols, aldehydes and terpenes, geosmin (52) was the common constituent to all isolates [26].

The production of the monoterpene linalool $(21 ; 38.53 \pm 5.19 \%)$ as major constituent by ACTB-77 called special attention in this work since similar result was found only in previous studies on VOCs produced by two strains of S. philanthi, RM-1-138 [16] and RL-1-178 [39], both isolated from the rhizosphere soil of chili peppers in Thailand. Among the $39 \mathrm{mVOCs}$ produced by RL-1-178 strain under axenic cultured in wheat seed medium, linalool $(\mathbf{2 1} ; 13.55 \%)$ and geosmin $(\mathbf{5 2} ; 13.75 \%)$ were also found as the major compounds [39]. Linalool $(\mathbf{2 1} ; 9.06 \%)$ was the main compound produced by RM-1-138 strain under the same experimental conditions, while geosmin (52) was found in only $1.23 \%$. 
S. clavuligerus linalool synthase (bLinS) was discovered and identified as catalysts for linalool production using metabolic engineering platform [40]. The use of bLinS in metabolically engineered monoterpene-producing E. coli strains yielded a 300fold higher linalool production compared with the corresponding linalool synthase from plant. Therefore, the identification of this monoterpene as major constituent produced by ACTB-77 and S. philanthi (RM-1-138 [16] and RL-1-178 [39] strains), may represent examples of linalool synthase expression in Streptomyces strains. It is worth highlighting that linalool has high commercial value since it is used in fragrance, cosmetic and non-cosmetic products, as well as intermediate in organic syntheses. Thus, the use of commercial linalool reaches more than 1,000 metric ton/year worldwide [40].

Comparison between VOCs produced by the actinobacteria ACTB-77 and those identified for the fungus $C$. musae, revealed compounds 3-methyl-butan-1-ol (1), 2-methyl-butan-1-ol (2) and $\beta$-myrcene (9) as the only chemical constituents common to both the microorganisms. Nevertheless, despite the alcohol 1 is a major compound produced by the fungus $(23.82 \pm 2.07 \%)$, it is found in only $1.77 \pm 0.11 \%$ in the actinobacteria VOCs composition. Both microorganisms produced $\beta$-myrcene (9) in low concentrations, $1.14 \pm 0.22 \%$ (C. musae) and $4.95 \pm 0.65$ (ACTB-77 strain).

\section{Actinobacteria Amycolatopsis sp. (ACTB-290)}

The study on VOCs produced by ACTB-290 strain under axenic culture provided 22 peaks recorded in the GC chromatogram (Table 2). From these, 18 peaks ( $82 \%$ of the peaks) were identified, accounting for $c a .95 \%$ of the total area of all recorded peaks. Dimethyl disulfide $(4 ; 48.49 \pm 3.48 \%)$ and dimethyl trisulfide $(\mathbf{8} ; 21.14 \pm 3.50 \%)$ were the major compounds, together representing $c a .70 \%$ of the VOCs composition. Besides these two, 2,4-dithiapentane $(6 ; 4.32 \pm 0.85 \%)$ and methyl(methylthio)methyl disulfide $(27 ; 1.95 \pm 0.07 \%)$ were also identified as sulfur-containing compounds. The 18 identified compounds were from five different groups, sulfur-derivatives (4 compounds; $c a$. $76 \%$ total area), alcohol (1 compound; $c a$. $1 \%$ total area), ketone ( 7 compounds; $c a .8 \%$ total area), ester ( 2 compounds; $c a .3 \%$ total area) and bicyclic monoterpene (4 compounds; $c a .7 \%$ total area).

Actinobacteria from Streptomyces and Amycolatopsis genera are known to be proficuous sources of non-volatile antibiotic compounds [41]. Nevertheless, opposite to Streptomyces that have VOCs profile reported from some of its species, no study on VOCs identification of Amycolatopsis species was found in the literature. It was clearly observed a significant difference between the VOCs profile of these two actinobacteria strains. While Streptomyces sp. (ACTB-77) produced terpenes (21 and 52) as main constituents, Amycolatopsis sp. (ACTB-290) yielded sulfur-containing compounds (4 and 8) as predominant in its VOCs composition. Herein, it is worth mentioning that, although dimethyl disulfide (4) and dimethyl trisulfide (8) were 
not produced by Streptomyces sp. (ACTB-77), they are included as two common constituents in the list of putative VOCs from eleven Streptomyces strains isolated from a Rhizoctonia-suppressive soil, besides a strain of S. lividans [26]. Additionally, comparison between VOCs profiles of actinobacteria ACTB-290 and C. musae showed no common compounds for these two microorganisms.

\section{mVOCs profile under co-culture}

Co-culture of ACTB-77 and C. musae

During the co-culture experiment for mVOCs extraction by HS-SPME it was clearly observed the inhibition of the fungus growing, corroborating the already discussed antifungal activity of ACTB-77 against $C$. musae. GC/MS analysis of the VOCs produced by the microorganisms in the co-culture experiment yielded 26 peaks (Table 2). Among them, only 3 compounds (ca. $1.7 \%$ of the total area) were not identified.

As already mentioned, compounds 3-methyl-butan-1-ol (1), 2-methyl-butan-1-ol (2) and $\beta$-myrcene (9) were the only VOCs produced by both microorganisms under axenic culture. These three compounds were also identified in the co-culture $(6.42 \pm 0.75,2.43 \pm 0.26$ and $1.33 \pm 0.02 \%$, respectively). Excluding these compounds, comparison of the VOCs profile of the actinobacteria under axenic culture (24 exclusive peaks) and that from the co-culture with the fungus revealed only ten compounds from ACTB-77. They are linalool $(\mathbf{2 1} ; 6.08 \pm 0.49 \%)$, $\alpha$-terpineol $(\mathbf{3 4} ; 1.68 \pm 0.08 \%)$, thymol methyl ether $(\mathbf{3 6}$; $0.95 \pm 0.05 \%)$, carvacrol methyl ether $(\mathbf{3 7} ; 0.64 \pm 0.03 \%)$, geraniol $\mathbf{( 3 8} ; 0.49 \pm 0.03 \%)$, geosmin $(\mathbf{5 2} ; 3.08 \pm 0.16 \%)$ and selina3,7(11)-diene (64; $1.43 \pm 0.12 \%)$, besides three non-identified (RT 12.082, 12.517 and $14.086 \mathrm{~min}$.). Therefore, fourteen VOCs produced by the actinobacteria under axenic culture were not detected in the co-culture experiment. It is worth highlighting that, $\mathbf{2 1}$ and 52, both major compounds produced by the actinobacteria under axenic culture, were found in much lower concentrations $(6.14 \pm 0.49 \%$ and $3.1 \pm 0.16 \%$, respectively) in the co-culture.

$\alpha$-Phellandrene $(\mathbf{1 2} ; 11.94 \pm 0.39 \%)$ and $\beta$-phellandrene $(\mathbf{1 5} ; 43.01 \pm 1.53 \%)$, both compounds identified as $C$. musae VOCs under the axenic culture $(8.09 \pm 1.74$ and $28.38 \pm 4.89 \%$, respectively), were the major constituents in the co-culture. In this latter experiment, a considerable increment on the production of 15 by the fungus was observed. Studies from the literature revealed the antibacterial activity of fruit and plant essential oils with high content of the sesquiterpene 15 [42,43]. Therefore, it is possible to expeculate that the increment of this compound in the co-culture may be a fungal defense. 

due to the presence of monoterpenes (especially linalool: 21) with the bioactivity already reported in the literature [44, $45,46,47,48]$.

Like the observed for the co-culture experiment of ACTB-77 and C. musae, during the VOCs extraction of ACTB-290 cocultured with the same fungus it was observed inhibition of the fungus growing. GC/MS analysis of the VOCs produced in the co-culture experiment recorded 36 peaks, with 31 of them $(86 \% ; c a .97 \%$ of the total area) being identified (Table 2). Through comparison of the peaks recorded in this experiment with those from the axenic cultures of the microorganisms, it was observed that 16 peaks ( 3 non-identified compounds) are related to compounds produced by the actinobacteria, 15 peaks were produced by the fungus, and 5 peaks ( 2 non-identified compounds) exclusive to the co-culture.

Among the 22 VOCs produced by the actinobacteria ACTB-290 cultured under axenic condition, only 2-methyl-2bornene (16), methyl(methylthio)methyl disulfide (27), 2-tetradecanone (65), (Z)-8-dodecen-1-ol acetate (66) and 1tetradecanol (67), besides one of the non-identified compounds (RT $10.87 \mathrm{~min}$.), were not observed in the co-culture. Concerning to C. musae, only compounds 3-methylbutan-1-ol acetate (5), p-menta-1,5-dien-8-ol (29) and aristolochene (59), which were produced by the fungus under axenic culture, were not detected in the co-culture. Again, the fungal VOCs $\alpha$ phellandrene $(12 ; 11.91 \pm 0.65 \%)$ and $\beta$-phellandrene $(15 ; 44.71 \pm 2.61 \%)$ were the major constituents in the co-culture. It is noteworthy the significant increasing of the fungal compound $\alpha$-terpinene $(\mathbf{1 3})$ concentration in the co-culture $(6.02 \pm 0.21 \%)$ when compared with the axenic culture $(3.43 \pm 0.74 \%)$.

Differently of what was observed in the co-culture of the fungus and the actinobacteria ACTB-77, the study involving strain ACTB-290 yielded five exclusive peaks. Among these, compounds with retention times of 14.503 and 14.805 min were not identified. The three identified mVOCs were 2-nonanol (23; $0.47 \pm 0.04 \%)$, 2-undecanol $(\mathbf{4 4} ; 0.65 \pm 0.05 \%)$ and 2-dodecanol $(50 ; 0.28 \pm 0.03 \%)$. Interestingly, these alcohols may be thought as bioreduction products of the respective $\mathrm{C}_{9}, \mathrm{C}_{11}$ and $\mathrm{C}_{12}$ ketones (VOCs from actinobacteria) by the fungus. Thus, the antifungal activity of Amycolatopsis sp. (ACTB-290) against $C$. musae may be associated to its sulfur-containing metabolites dimethyl disulfide (4) and dimethyl trisulfide (8), both previously reported as potent fungicides [49, 50]. 


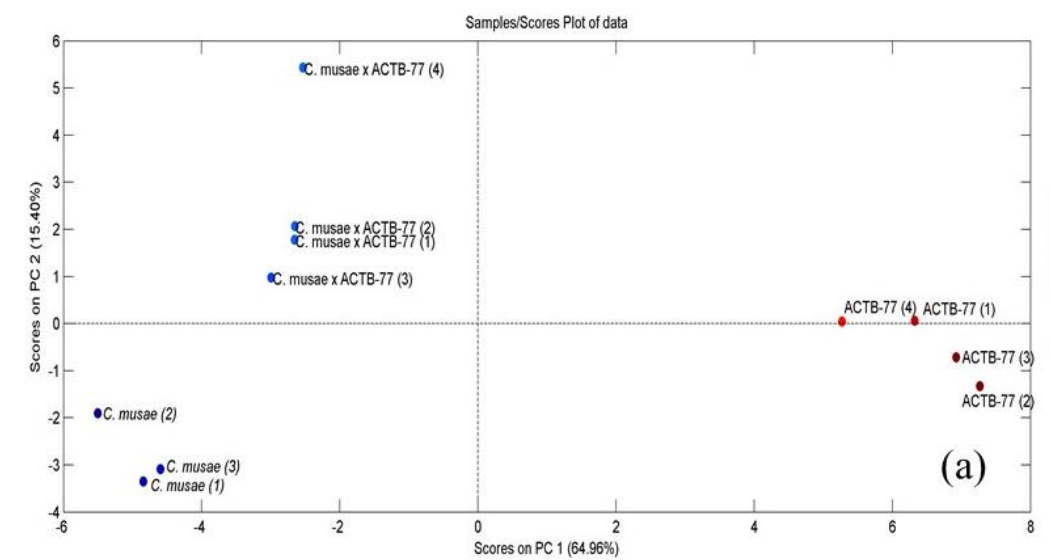

\section{ACTB-77, C. musae and their co-culture} the actinobacteria (ACTB-77), C. musae and co-culture, respectively.

The statistical analysis discussed herein is related to the comparison between the relative concentrations of the peak areas of each compound produced by the microorganisms under axenic culture and those produced in the co-culture (ACTB-77 and $C$. musae; ACTB-290 and C. musae). A principal component analysis (PCA) [51] was performed, which is an unsupervised partner recognition algorithm used to represent a high number of results through two graphics called scores and loadings. The scores graph presents the similarity/differences between each culture while the loadings graphic displays the constituent that is important for differences and similarities between each group. In summary, all peaks are converted into new variables, called PCs, each one accounting for the data variability. Thus, similar samples are close in the scores graphic while different samples are distant. The relative concentration of each constituent is displayed in loadings graphics, which have the same axes presented in the scores graphics. When the scores and loadings graphics are overlaid, it is possible to deduce that the relative concentration of a constituent will be higher for the sample with high scores. Each number displayed in loadings graphics represents a peak which it is identified in Table 2, and which was listed in increasing order of the retention index.

PC1 and PC2 explain $80.36 \%$ of the data variance (Figure 6), being possible to analyze all constituents in the samples using only these two variables. It was observed a natural separation tendency of the groups, which are represented by red (ACTB77 strain), dark blue (C. musae strain) and light blue (co-culture) colors (Figure 6a). In this case, the highest separation was observed for the samples from the axenic culture of the actinobacteria since these samples presented the highest scores values in PC1 (Figure 6a). The relative concentrations of the constituents are represented in the loadings graph (Figure 6b) by circles with three different colors, where red, dark blue and light blue are associated to the highest concentration constituents from

Figure 6. Scores (a) and loadings (b) graphs obtained by PC1 and PC2 for the samples of ACTB-77 (red), C. musae (dark blue) and the co-culture (light blue). 
Figure 6b shows 3-methyl-butan-1-ol (1), 2-methyl-butan-1-ol (2), $\alpha$-phellandrene (12), $\beta$-phellandrene (15), phenylethyl alcohol (24), p-mentha-1,5-dien-8-ol (29), menthol (31) and aristolochene (59) as constituents from the C. musae group with the highest relatively concentrations. Among them, compounds 1, 12, 15, 24 and $\mathbf{5 9}$ represent $c a$. 75\% of the VOCs composition of the fungus. Concerning the constituents from the ACTB-77 group, geraniol (38), 1-decanol (41), 2-undecenal (47), geosmin (52), $\gamma$-gurjunene (58) and mint sulfide (69) are displayed in Figure $\mathbf{6 b}$ as those mVOCs with the highest relatively concentrations. Although $\mathbf{3 8}$ and $\mathbf{5 2}$ are found in both co-culture and ACTB-77 axenic culture, these constituents are represented in higher concentrations in the latter experiment. It worth highlighting that $\alpha$-phellandrene (12), $\beta$-phellandrene (15) and silphinene (46), already found in axenic culture of $C$. musae, had their relative concentrations intensified in the coculture experiment (Figure 6b).

PC1 scores were not significant to differentiate samples of $C$. musae and co-culture, since it is possible to observe a correlation between the variables highlighted in blue (dark and light) in the loadings graph (Figure 6b). It is noteworthy that 3-methyl-butan-1-ol (1) and $\beta$-phellandrene (15), both found as main VOCs produced by C. musae, are the most important constituents for distinguishing the fungus from the co-culture. These two chemical constituents are not correlated, and they present significant difference in their relative concentrations in the two groups ( C. musae and co-culture). The differentiation between $C$. musae and co-culture groups can be observed through the different values of their PC2 scores (Figure 6a).

\section{ACTB-290, C. musae and their co-culture}

Together, PC1 and PC2 explain 76.84\% of the data variance (Figure 7), making possible the analysis of similarities/differences of the groups using only these two variables. Comparison of this study with the previously discussed for the experiments involving actinobacteria ACTB-77 revealed a higher separation tendency of the groups for the studies with ACTB-290, that are represented by dark blue (ACTB-290 strain), dark red (C. musae strain) and three different colors (light and dark orange; green) for co-culture (Figure 7a). The use of three colors for co-culture experiments were needed to indicate different composition in their replicates.
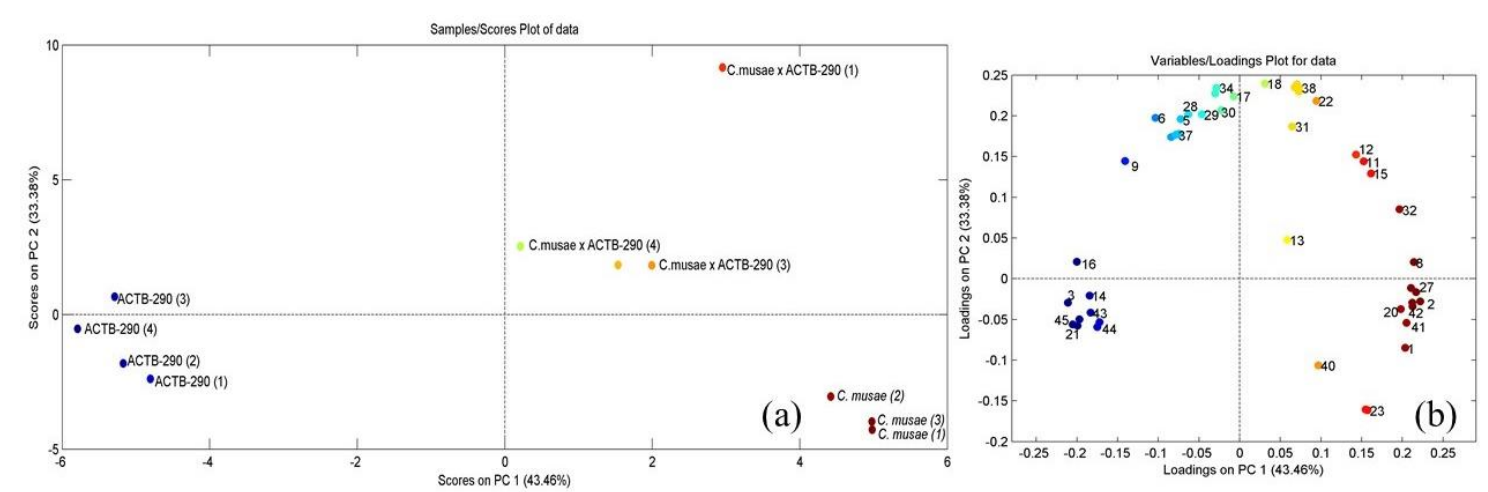
Figure 7. Scores (a) and loadings (b) graphs obtained by PC1 and PC2 for the samples of ACTB-290 (dark blue), C. musae (dark red) and the co-culture (light orange, dark orange and green).

As observed in the loadings graph (Figure 7b), the lowest scores of PC1 are the variables highlighted in dark blue, which are related to the axenic culture of ACTB-290. In this graph, dimethyl disulfide (4), dimethyl trisulfide (8) and methyl 2ethylhexanoate (18), Table 2, are significant variables to distinguish ACTB-290 from the other two groups (C. musae and coculture). Additionally, these three chemical constituents are found in relatively higher concentrations in the actinobacteria axenic culture when comparing with the co-culture. $\alpha$-Phellandrene (12), $\alpha$-terpinene (13) and $\beta$-phellandrene (15), Table 2, which are represented in the loadings graph (Figure 7b) with dark red circles, present positive values of scores in PC2. This agrees with the fact of these mVOCs had a significant increasing in the co-culture experiment when compared to the axenic culture of the fungus.

As already mentioned, the sulphur-containing compounds dimethyl disulfide (4), dimethyl trisulfide (8) and methyl(methylthio)methyl disulfide (27), Table 2, all VOCs exclusively produced by the actinobacteria ACTB-290, are reported as potent fungicides. Interestingly, no correlation between these compounds and the alcohols $(\mathbf{2 3}, \mathbf{4 4}$ and $\mathbf{5 0}$, Table 2) recorded exclusively in the co-culture experiment was observed in the correlation map (Figure 5S). This suggest that these alcohols are products of bioreduction of actinobacteria produced ketones 22,43 and 48 , respectively, by fungus strain.

Global analysis

A global analysis of the aforementioned experiments was performed since, opposite to a non-statistical approach, PCA allows to evaluate all samples (ACTB-77, ACTB-290, fungus and co-cultures) grouped in the same graphs (Figure 8). In this case, PC1, PC2 and PC3 explain $75.83 \%$ of the data variance, allowing the analysis of similarities/differences of the groups using these three variables. It was observed a high separation tendency of the groups, that are represented by dark red (ACTB-77), dark blue (ACTB-290), cyan (C. musae) and green (ACTB-77/C. musae) and light blue (ACTB-290/C. musae) colors in the scores graphs depicted in Figure 8a (PC1 vs. PC2) and Figure 8b (PC1 vs. PC3). 

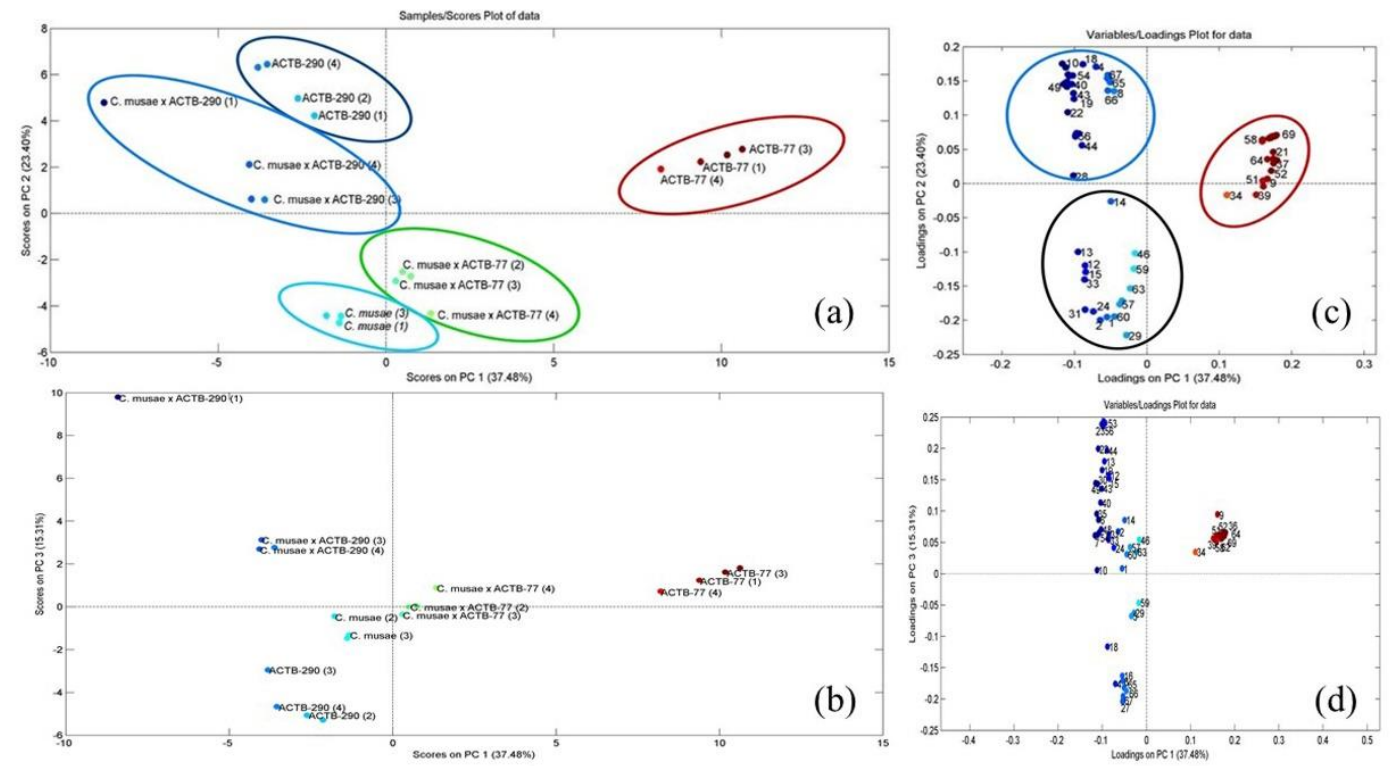

Figure 8. Scores (a) and loadings (b) graphs obtained by PC1 and PC2 for the samples ACTB-77 (dark red), ACTB-290 (dark blue), C. musae (cyan) and co-cultures ACTB-77/C. musae (green) and ACTB-290/C. musae (light blue).

As observed in Figures 8a and $\mathbf{8 b}$, co-culture ACTB-77/C. musae has greater similarity with the fungus group than with the actinobacteria group. Concerning the co-culture involving the actinobacteria ACTB-290 (ACTB-290/C. musae), it was observed greater similarity with the actinobacteria group than the fungus group. These suggest a more pronounced antifungal activity for ACTB-290 strain, which agrees with the SEM images of the fungus filaments after its inhibition by ACTB-290 previously discussed.

The loadings graphs displayed in Figure 8c (PC1 vc. PC2) and Figure 8d (PC1 vc. PC3) show that mVOCs inside the red circle (Figure 8c) present significantly higher concentration for ACTB-77 samples when compared with the other groups, besides higher loadings values in PC1. The mVOCs inside the blue circle present a significant higher concentration for both ACTB-290 and the co-culture (ACTB-290/C. musae), indicating that these two groups present similar mVOCs.

Dimethyl disulfide (4), (S)-verbenone (10), 2-undecanone (43) and 2-tridecanone (54), Table 2, are present in significatively lower concentration in the co-culture ACTB-290/C. musae when compared with the axenic culture of the actinobacteria

(Figures 8c and 8d). Ketones $\mathbf{4 3}$ and $\mathbf{5 4}$ are those produced exclusively by the actinobacteria that were reduced by the fungus, while dimethyl disulfide (4) is a potent fungicide. constituents numbered according to Table 2 . The correlation is calculated pair by pair and represented by squares in the map, 
in which dark blue means an inverse correlation $(\mathrm{r}=-1)$ and dark red means a direct correlation $(\mathrm{r}=+1)$. The closer to $+1 /$ 1become, the higher the correlation between the peaks will be. Analysis of this map reveals that ketones $\mathbf{2 2}, \mathbf{4 3}$ and $\mathbf{5 4}$, which were suggested to be reduced by the fungus, present an inverse correlation with their respective alcohols 23,44 and 50 (Table 2), confirming the biotransformation occurrence in the co-culture. In addition, there is a slightly positive correlation between these ketones and the sulphur-containing compound 4, suggesting that when the ketones concentrations reduce, the concentration of $\mathbf{4}$ also reduces.

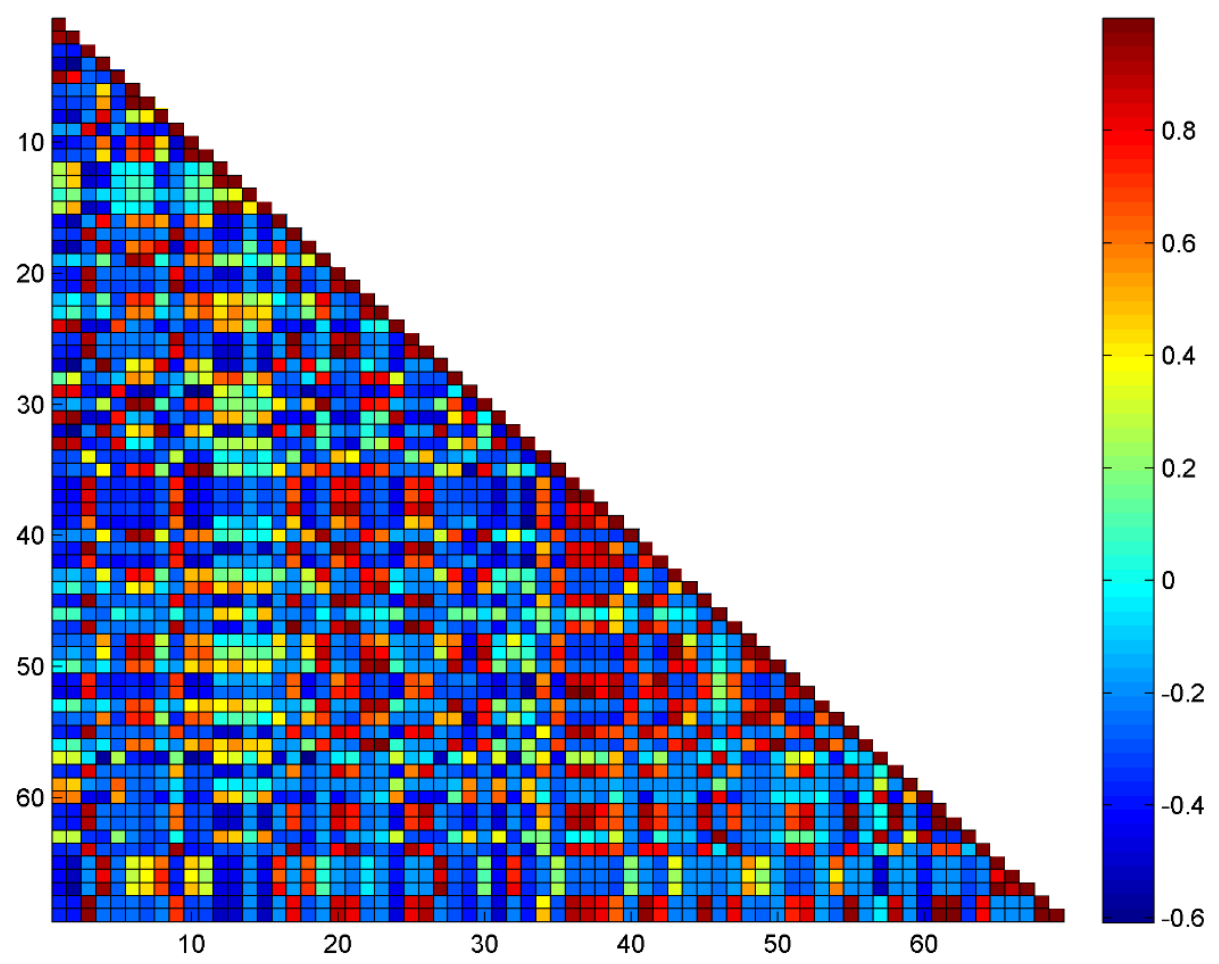

Figure 9. Correlation map for VOCs of the samples ACTB-77, ACTB-290, C. musae and co-cultures ACTB-77/C. musae and ACTB-290/C. musae. The correlation goes from -1 (dark blue) to 1 (dark red) scale and it was calculated pair by pair. against $C$. musae and rise the hypothesis that the sulphur-containing constituents are responsible for the bioactivity.

\section{Conclusion}

In summary, rhizosphere soil of plants from Caatinga biome was shown to be a source of actinobacteria strains that produce volatile organic compounds with antifungal activity against phytopathogen $C$. musae. Among the strains, the most active were 
Streptomyces sp. (ACTB-77) and Amycolatopsis sp. (ACTB-290). The latter presented the highest growing inhibition of the fungus, a behavior corroborated by the greatest damage of its VOCs on the fungal hyphae morphology. HS-SPME-GCMS analyses of VOCs produced by ACTB-77 and ACTB-290, revealed linalool and geosmin as major constituents for ACTB-77, and dimethyl disulfide and dimethyl trisulfide as major VOCs compounds produced by ACTB-290. The sulphur-containing VOCs produced by ACTB-290 are known as potent antifungal compounds, therefore justifying the more pronounced activity of this strain. Additionally, statistical analyses of the experiments revealed that sulphur-containing VOCs are significant variables to distinguish ACTB-290 from C. musae and the co-culture.

\section{Acknowledgement}

The authors are thankful to the Coordenação de Aperfeiçoamento de Ensino Superior (CAPES) for the sponsorships of M. V. Brito (Process: 88887.319063/2019-00) and M. S. Netto (Process: 88887.479095/2020-00), and financial support (Finance Code 001- PROEX 23038.000509/2020-82. No AUXPE: 1227/2020). M. C. F. de Oliveira (Process: 310881/2020-0) and M. C. de Mattos (Process: 306043/2018-1) thank to Conselho Nacional de Desenvolvimento Científico e Tecnológico (CNPq) for their research sponsorships. L. Pinto thank the Brazilian funding agency Fundação de Amparo a Ciência e Tecnologia do Estado de Pernambuco (FACEPE 14/2019 - INOVA IAM; Process: APQ-0437-1.06/19).

\section{References}

1. Harir M, Bendif H, Bellahcene M, Zohra F, Rebecca P (2018) Streptomyces Secondary Metabolites. Basic Biology and Applications of Actinobacteria 6:100-122. https://doi.org/10.5772/intechopen.79890

2. David B, Wolfender JL, Dias DA (2015) The pharmaceutical industry and natural products: historical status and new trends. Phytochemistry Reviews 14:299-315. https://doi.org/10.1007/s11101-014-9367-z

3. Schulz-Bohm K, Martín-Sánchez L, Garbeva P (2017) Microbial Volatiles: Small Molecules with an Important Role in Intra and Inter-Kingdom Interactions. Front. Microbiol 8:2484. https://doi.org/10.3389/fmicb.2017.02484

4. Mari M, Bautista-Baños S, Sivakumar D (2016) Decay control in the postharvest system: Role of microbial and plant volatile organic compounds. Postharvest biology and technology 122:70-81. https://doi.org/10.1016/j.postharvbio.2016.04.014 
5. Choudoir M, Rossabi S, Gebert M, Helmig D, Fierer N (2019) A phylogenetic and functional perspective on volatile organic compound production by Actinobacteria. mSystems 4:e00295-18. https://doi.org/10.1128/mSystems.0029518

6. Ross C, Opel V, Scherlach K, Hertweck C (2014) Biosynthesis of antifungal and antibacterial polyketides by Burkholderia gladioli in coculture with Rhizopus microspores. Mycoses 57: 1-8. https://doi.org/10/1111/myc.12246

7. Brakhage AA, Schoroeckh V (2011) Fungal secondary metabolites strategies to activate silent gene clusters. Fungal Genetics and Biology 48:15-22. https://doi.org/10.1016/j.fgb.2010.04.004

8. Bertrand S, Bohni N, Schnee S, Schumpp O, Gindro K, Wolfender, JL (2014) Metabolite induction via microorganism co-culture: a potential way to enhance chemical diversity for drug discovery. Biotechnology Advances 32:1180-1204. https://doi.org/10.1016/j.biotechadv.2014.03.001

9. Bertrand S, Schumpp O, Bohni N, Monod M, Gindro K, Wolfender JL (2013) De novo Production of Metabolites by Fungal co-culture of Trichophyton rubrum and Bionectria ochroleuca. Journal of Natural Products 76:1157-1165. https://. doi.org/10.1021/np400258f

10. Deng X, Zhang N, Shen Z, Zhu C, Li R, Salles JF, Shen Q (2019) Rhizosphere bacteria assembly derived from fumigation and organic amendment triggers the direct and indirect suppression of tomato bacterial wilt disease. Applied soil ecology 30:1-9. https://doi.org/10.1016/j.apsoil.2019.103364

11. Bonfante P, Anca I A (2009) Plants, Mycorrhizal Fungi, and Bacteria: A Network of Interactions. Annual Review of Microbiology 63: 363-383. https://doi.org/0066-4227/09/1013-0363

12. Yadav AK, Srivastava A K, Yandigeri MS, Kashyap SK, Modi DR, Arora DK (2010) Characterization of indigenous copper-resistant Streptomyces from chickpea (Cicer arietinum L.) fields. Annals of Microbiology 60: 605-614. https://doi.org/10.1007/s13213-010-0095-3

13. Sharma M, Dangi p, Choudhary M (2014) Actinomycetes: Source, Identification, and Their Applications. International Journal of Current Microbiology and Applied Sciences 3:801-832

14. Scholler CEG, Gurtler H, Pedersen R, Molin S, Wilkins K (2002) Volatile metabolites from actinomycetes. Journal of agricultural and food chemistry 50: 2615-2621. https://doi.org/10.1021/jf0116757

15. Sharma V, Salwan R (2018) Biocontrol potential and applications of actinobacteria in agriculture. In: New and future developments in microbial biotechnology and bioengineering. Elsevier, India, pp 93-108. https://doi.org/10.1016/B978-0-444-63994-3.00006-0

16. Boukaew S, Plubrukam A, Prasertsan P (2013) Effect of volatile substances from Streptomyces philanthi RM-1-138 on growth of Rhizoctonia solani on rice leaf. BioControl 58: 471-482. https://doi.org/10.1007/s10526-013-9510-6 
17. Li Q, Ning P, Zheng L, Huang J, Li G, Hsiang T (2012) Effects of volatile substances of Streptomyces globisporus JK-1 on control of Botrytis cinerea on tomato fruit. Biological Control 61:113-120. https://doi.org/10.1016/j.biocontrol.2011.10.014

18. Nzekoue FK, Caprioli G, Fiorini D, Torregiani E, Vittori S, Sagratin G (2019) HS-SPME-GC-MS technique for FFA and hexanal analysis in different cheese packaging in the course of long term storage. Food Research International

22. Oliveira FC, Barbosa FG, Mafezoli J, Oliveira MCF, Camelo ALM, Longhinotti E, Lima ACA, Câmera MPS, Gonçalves FJT, Freire FCO (2015) Volatile Organic Compounds from Filamentous Fungi: a Chemotaxonomic Tool of the Botryosphaeriaceae Family. Journal of the Brazilian Chemical Society 26:2189-2194. https://doi.org/10.5935/0103-5053.20150204

23. Lazazzara V, Perazzolli M, Pertot I, Biasioli F, Puopolo G, Cappellin L (2017) Growth media affect the volatilome and antimicrobial activity against phytophthora infestans in four Lysobacter type strains. Microbilogival Reseach 201: 52-62. https://doi.org/10.1016/j.micres.2017.04.015

24. Amini J, Agapoor Z, Ashengroph M (2016) Evaluation of Streptomyces spp. against Fusarium oxysporum f. sp. ciceris for the management of chickpea wilt. Journal of Plant Protection Research 56(3): 257-254. https://doi.org/10.1515/jppr-2016-0038

25. Zhang Q, Zhang J, Yang L, Zhang L, Jiang D, Chen W, Li G (2014) Diversity and biocontrol potential of endophytic fungi in Brassica napus. Biological control 72: 98-108. http://doi.org/10.1016/j.biocontrol.2014.02.018 
26. Cordovez V, Carrion VJ, Etalo DW, Mumm R, Zhu H, Wezil GPV, Raaijmakers J.M (2015) Diversity and functions of volatile organic compounds produced by Streptomyces from a disease-suppressive soil. Frontiers in Microbiology 6:1-13. https://doi.org/ 10.3389/fmicb.2015.01081

27. Kumar S, Stecher G, Tamura K (2016) MEGA7: molecular evolutionary genetics analysis version 7.0 for bigger datasets. Molecular Biology and Evolution 33(3): 1870-1874. https://doi.org/10.1093/molbev/msw054

28. Hall $\mathrm{T}$ (2012) BioEdit v7.2.5: Biological sequence alignment editor for Win95/98/2K/XP/7.http://www.mbio.ncsu.edu/bioedit/bioedit.html. Accessed 13 September 2018.

29. Sawoszczuk T, Sygula-Cholewinska J, Hoyo-Meléndez JMD (2015) Optimization of headspace solid phase microextraction for the analysis of microbial volatile organic compounds emitted by fungi: application to historical objects. Journal of chromatography A 1409:30-45. https://doi.org/10.1016/j.chroma.2015.07.059

30. Adams RP (2017) Identification of essential oil components by gas chromatography/mass spectrometry. Allured publishing, Carol Stream-USA

31. Mookherjee A, Bera P, Mitra A, MAITI MK (2017) Characterization and synergistic effect of antifungal volatile organic compounds emitted by the Geotrichum candidum PF005 an endophytic fungus from the eggplant. Microbial Ecology 75:647-661. https://doi.org/10.1007/s00248-017-1065-0

32. Yang M, Lu L, Pang J, Hu Y, Guo Q, Li Z, Wu S, Liu H, Wang C (2019) Biocontrol activity of volatile organic compounds from Streptomyces alboflavus TD-1 against Aspergillus flavus growth and aflatoxin production. Journal of microbiology 57(5):396-404. https://doi.org/10.1007/512275-019-8519-9

33. Vilaplana R, Pazmiño L, Valencia-Chamorro S (2018) Control of anthracnose, caused by Colletotrichum musae, on postharvest organic banana by thyme oil. Postharvest Biology and Technology 138:56-63. https://doi.org/10.1016/j.postharvbio.2017.12.008

34. Couto EF, Menezes M (2005) Physiomorphological characterization of Colletotrichum musae isolates. Brazilian Phytopathology 29:406-412. https://doi.org/10.1590/s0100-41582004000400008

35. Jayakumar V, Ramesh SA, Viswanathan R (2020) Biocontrol of Colletotrichum falcatum with volatile metabolites produced by endophytic bacteria and profiling VOCs by headspace SPME coupled with GC-MS. Sugar Tech. doi:10.1007/s12355-020-00891-2

36. Zhao P, Li P, Wu S, Zhou M, Zhi R, Gao H (2019) Volatile organic compounds (Vocs) from Bacillus subtiles CF-3 reduce anthracnose and elicit active defense responses in harvested litchi fruits. AMB Express 9:119. https://doi.org/10.1186/s13568-019-0841-2 
37. Quintana-Rodriguez E, Morales-Vargas AT, Molina-Torres J, Ádame-Alvarez RM, Acosta-Gallegos JA, Heil M (2014) Plant volatiles cause direct, induced and associational resistance in common bean to the fungal pathogen Colletotrichum lindemuthianum. Journal of Ecology 103(1):250-260. https://doi.org/10.1111/1365-2745.12340

38. Rojas-Flores C, Ventura-Aguilar RI, Bautista-Baños S, Revah S, Saucedo-Lucero JO (2019) Estimating CO2 and VOCs production of Colletotrichum fragariae and Rhizopus stolonifer grown in cold stored strawberry fruit. Microbiological Research. https://doi.org/10.1016/j.micres.2019.126327

39. Boukaew S, Prasertsan P (2020) Efficacy of volatile compounds from Streptomyces philanthi RL-1-178 as a biofumigant for controlling growth and aflatoxin production of the two aflatoxin-producing fungi on stored soybean seeds. Journal of Applied Microbiology. https://doi.org/10.1111/jam.14643

40. Karuppiah V, Ranaghan KE, Leferink NGH, Johannissen LO, Shanmugam M, Cheallaigh AN, Bennett NJ, Kearsey LJ, Takano E, Gardiner JM, Van der Kamp MW, Hay S, Mulholland AJ, Leys D, Scrutton NS (2017) Structural Basis of Catalysis in the Bacterial Monoterpene Synthases Linalool Synthase and 1,8-Cineole Synthase. ACS Catalysis 7(9): 6268-6282. https://doi.org/10.1021/acscatal.7b01924

41. Zhao P, Xue Y, Gao W, Li J, Zu X, Fu D, Feng S, Bai X, Zuo Y, Li P (2018) Actinobacteria-Derived Peptide Antibiotics since 2000. Peptides 103:48-59. https://doi.org/10.1016/j.peptides2018.03.011

42. Lan-Phi NT, Vy, T T (2015) Chemical composition, antioxidant and antibacterial activities of peels'essential oils of different pomelo varieties in the south of Vietnam. International Food Research Journal 22(6): 2426-2431

43. Mohammadhosseini M (2012) Chemical Profile and Antibacterial Activity in Hydrodistilled Oil from Aerial Parts of Prangos ferulacea (L.) Lindl. and Prediction of Gas Chromatographic Retention Indices by Using Genetic Algorithm Multiple Linear Regressions. Asian Journal of Chemistry 24(9): 3814-3820

44. Oliveira LMI, Araújo MAC, Souza SKV, Cardoso GN, Oliveira LE, Oliveira PF. (2017) Investigation of the antifungal potential of linalool against clinical isolates of fluconazole resistant Trichophyton rubrum. Journal de Mycologie Médicale 27(2):195-202. https://doi.org/10.1016/j.mycmed.2017.01.011

45. Dias IJ, Trajano ERIS, Castro RD, Ferreira GLS, Medeiros HCM, Gomes DQC (2017) Antifungal activity of linalool in cases of Candida spp. isolated from individuals with oral candidiasis. Brazilian Journal of Biology 78(2):368374. https://doi.org/10.1590/1519-6984.171054

46. Zhou H, Tao N, Jia L (2014) Antifungal activity of citral, octanal and $\alpha$-terpineol against Geotrichum citri-aurantii. Food Control 37:277-283. http://doi.org/10.1016/j.foodcont.2013.09.057 
47. Lira MHP, Andrade Júnior FP, Moraes GFQ, Macena GS, Pereira FO, Lima IO (2020). Antimicrobial activity of geraniol: an integrative review. Journal of Essential Oil Research 111. http://doi.org/10.1080/10412905.2020.1745697

48. Elshafie HS, Mancini E, Sakr S, De Martino L, Mattia CA, De Feo V, Camele I (2015) Antifungal Activity of Some Constituents of Origanum vulgare L. Essential Oil Against Postharvest Disease of Peach Fruit. Journal of Medicinal Food 18(8): 929-934. https://doi.org/ 10.1089/jmf.2014.0167

49. Wang C, Wang Z, Qiao X, Li Z, Li F, Chen M, Wang Y, Huang Y, Cui H (2013) Antifungal activity of volatile organic compounds from Streptomyces alboflavusTD-1. FEMS Microbiology Letters 341(1):45-51. https://doi.org/10.1111/1574-6968.12088

50. Wang Z, Zhong T, Chen K, Du M, Chen G, Chen X, Wang K, Zalan Z, Takacs K, Kan J (2020) Antifungal activity of volatile organic compounds produced by Pseudomonas fluorescens ZX and potential biocontrol of blue mold decay on postharvest citrus. Food Control. https://doi.org/10.1016/j.foodcont.2020.107499

51. Bro R, Smilde AK (2014) Principal component analysis. Anal. Methods 6(9):2812-2831. 
Figures

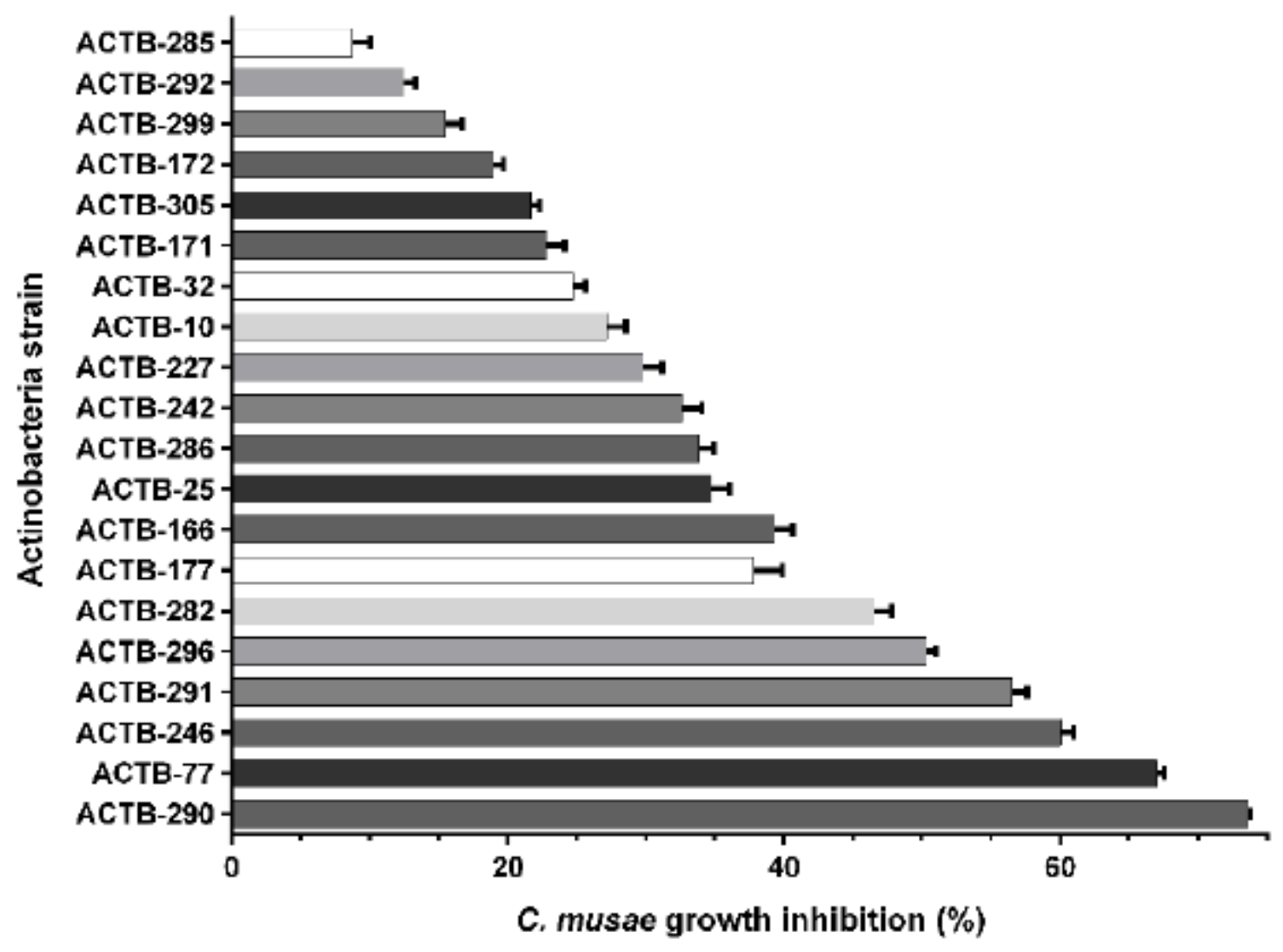

Figure 1

Growth inhibition (\%) of C. musae by volatile organic compounds (VOCs) produced by the twenty actinobacteria strains assayed. 


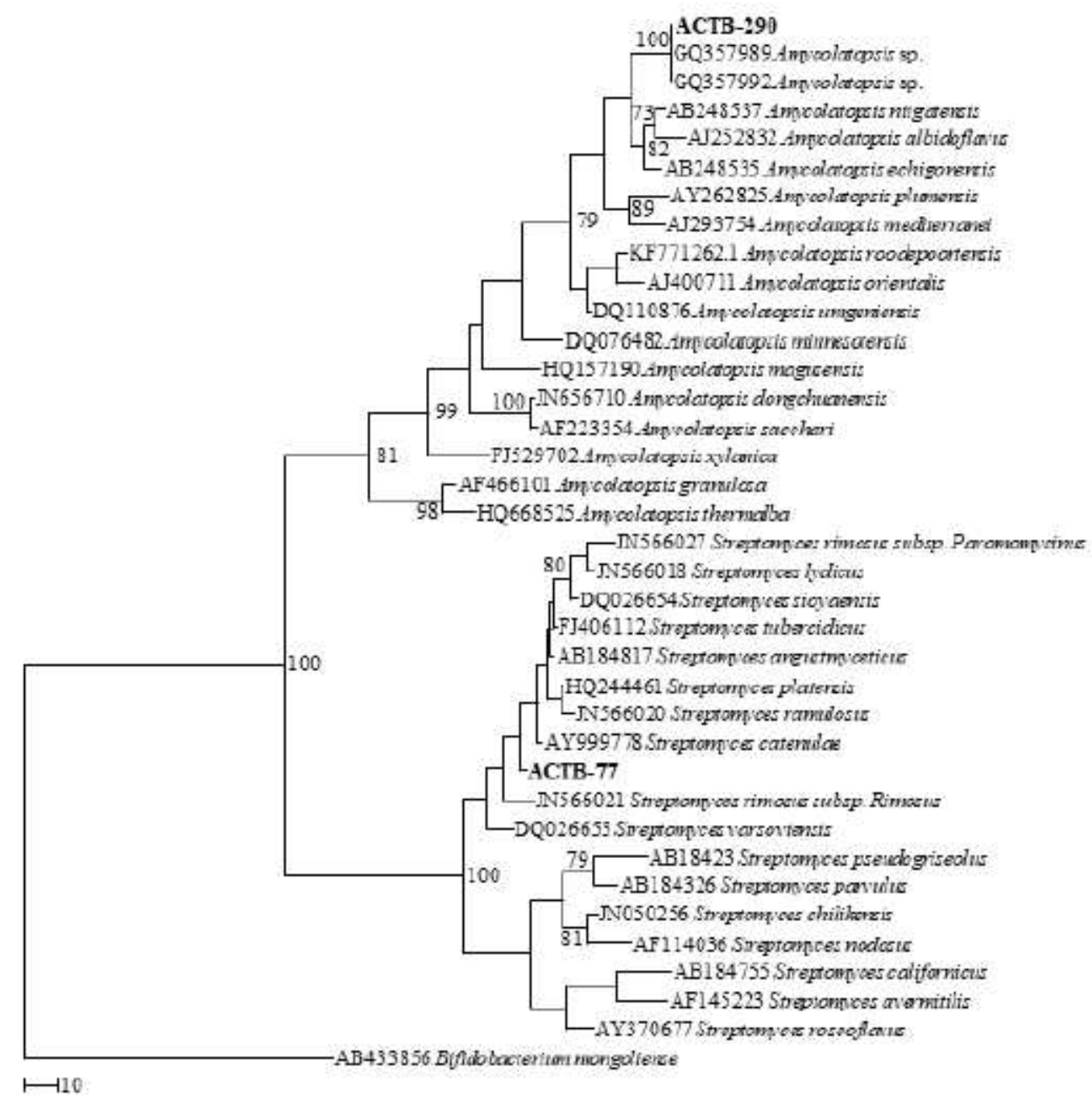

\section{Figure 2}

Phylogenetic tree inferred by maximum parsimony (MP) of data from the $16 \mathrm{~S}$ genomic region of the rDNA for sequences of the genera Amycolatopsis and Streptomyces. Bootstrap values ( $>70 \%)$ with 1000 repetitions are shown in the respective branch. Bifidobacterium mongoliense was used as an external group. The sequences in this study are highlighted in bold. 


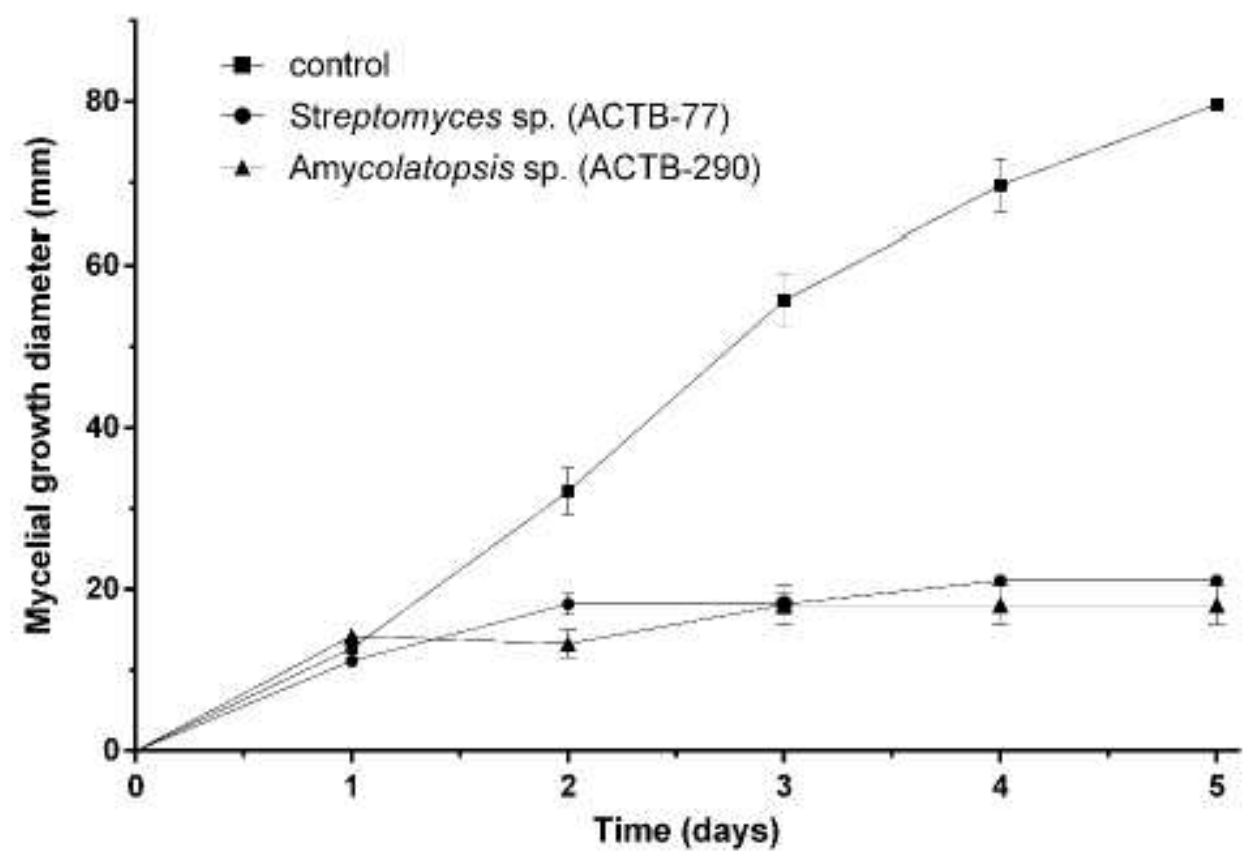

Figure 3

Results from the antifungal activity of Streptomyces sp. (ACTB-77) and Amycolatopsis sp. (ACTB-290) against C. musae.
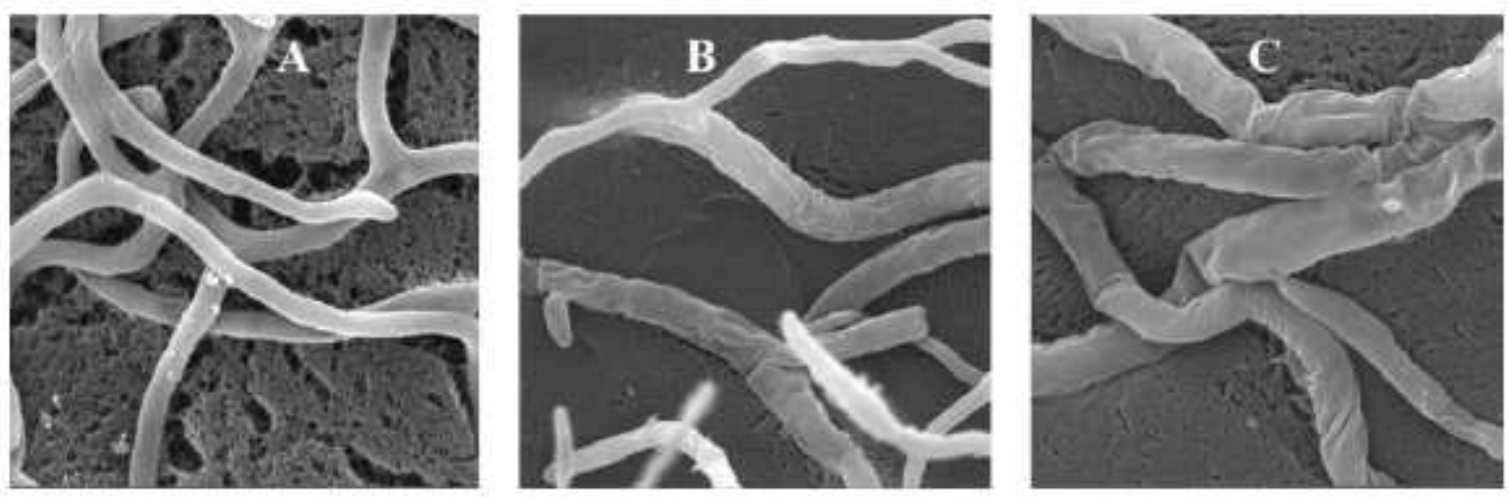

Figure 4

SEM imaging $(20 \mu \mathrm{M})$ of $\mathrm{C}$. musae hyphae in the fifth day of experiment: (A) under axenic culture (control); (B) in co-culture with Streptomyces sp.; (C) in co-culture with Amycolatopsis sp. 

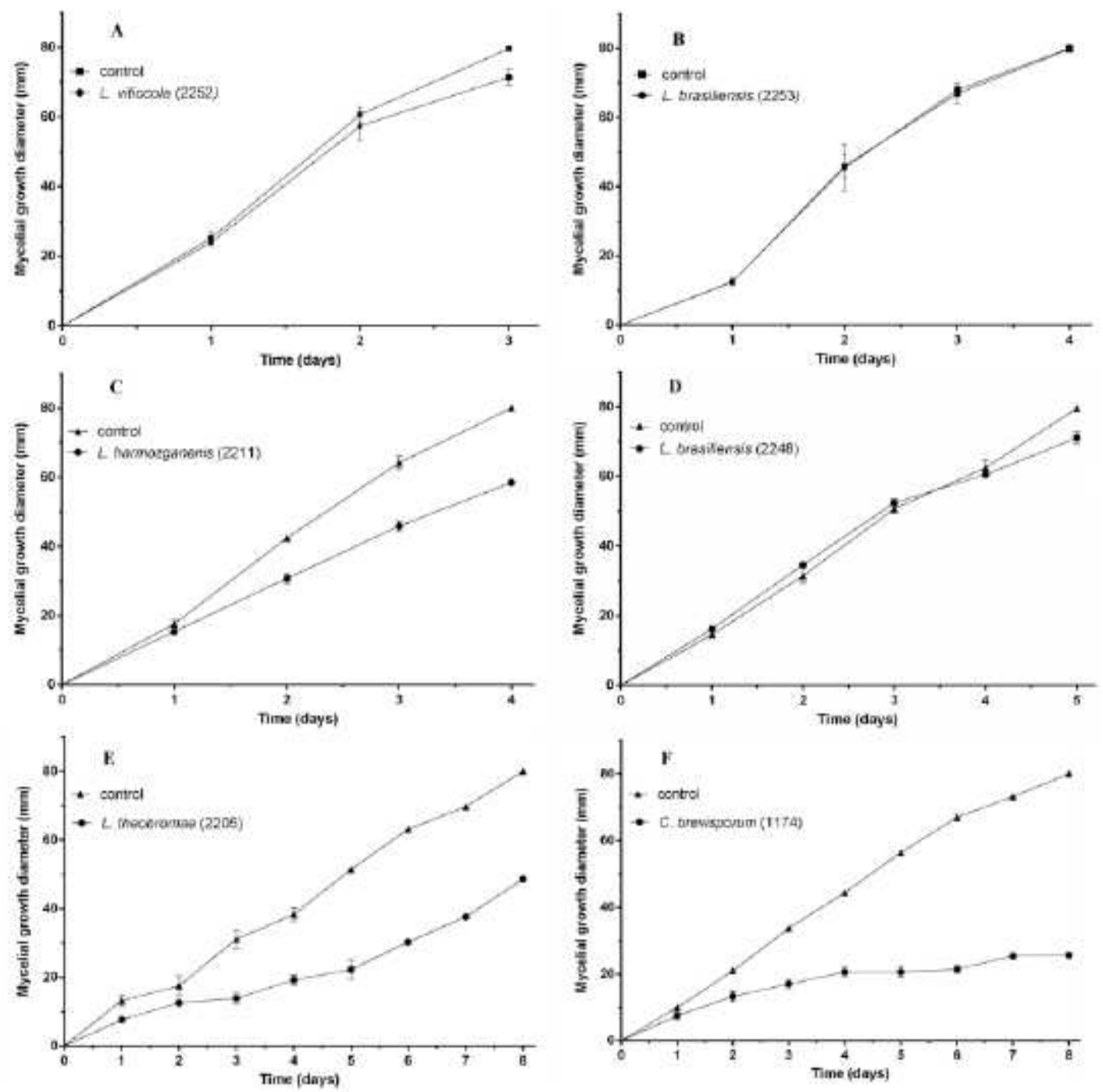

Figure 5

Results from the antifungal activity of Amycolatopsis sp. (ACTB-290) against: (A) Lasiodiplodia viticola; (B) L. brasiliensis-2253; (C) L. brasiliensis-2248; (D) L. theobromae; (E) Colletotrichum brevisporum. Fungus strain under axenic culture is the control experiment. 

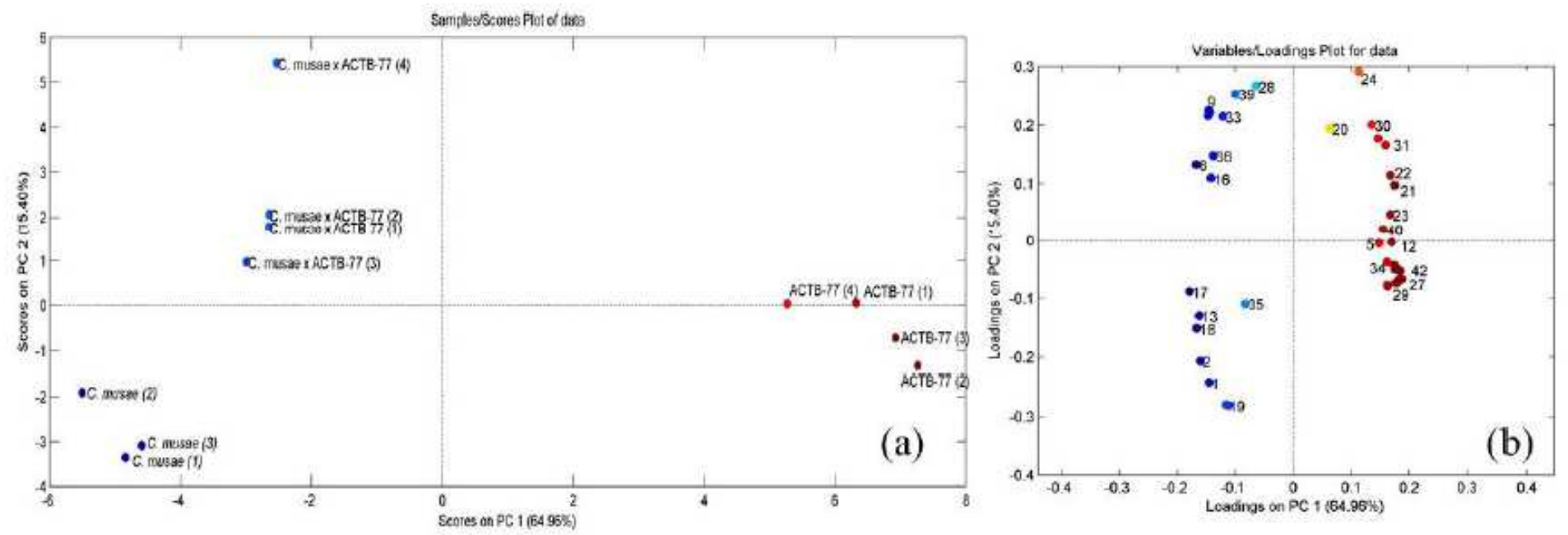

Figure 6

Scores (a) and loadings (b) graphs obtained by PC1 and PC2 for the samples of ACTB-77 (red), C. musae (dark blue) and the co-culture (light blue).
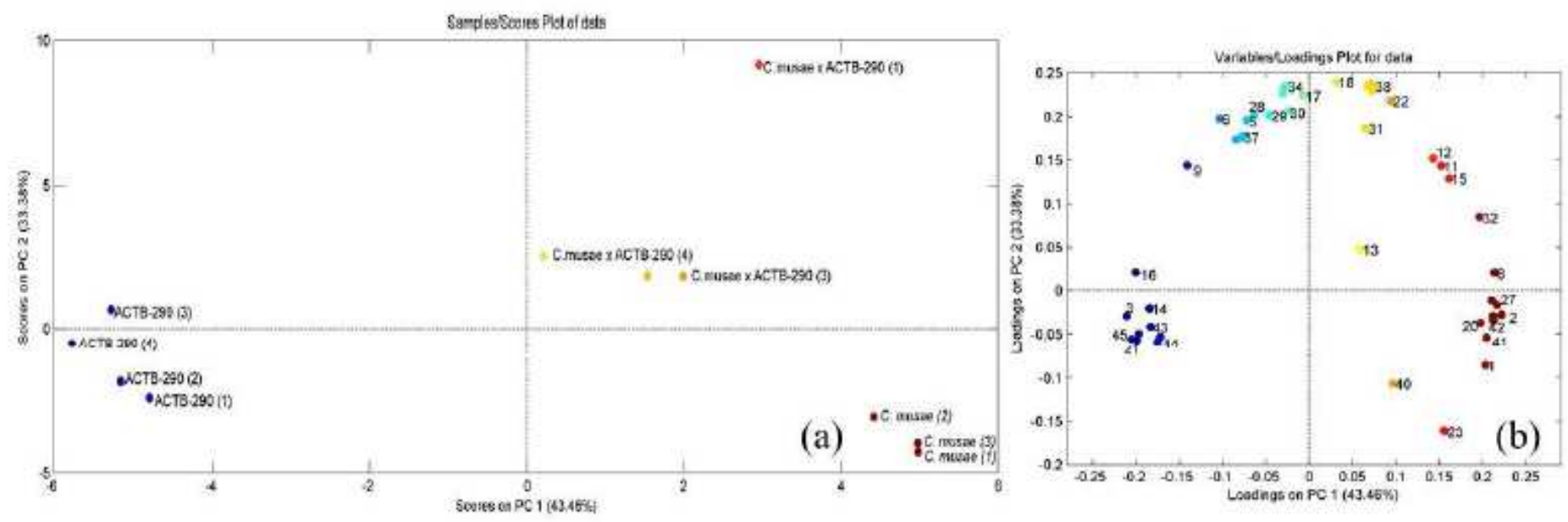

\section{Figure 7}

Scores (a) and loadings (b) graphs obtained by PC1 and PC2 for the samples of ACTB-290 (dark blue), C. musae (dark red) and the co-culture (light orange, dark orange and green). 

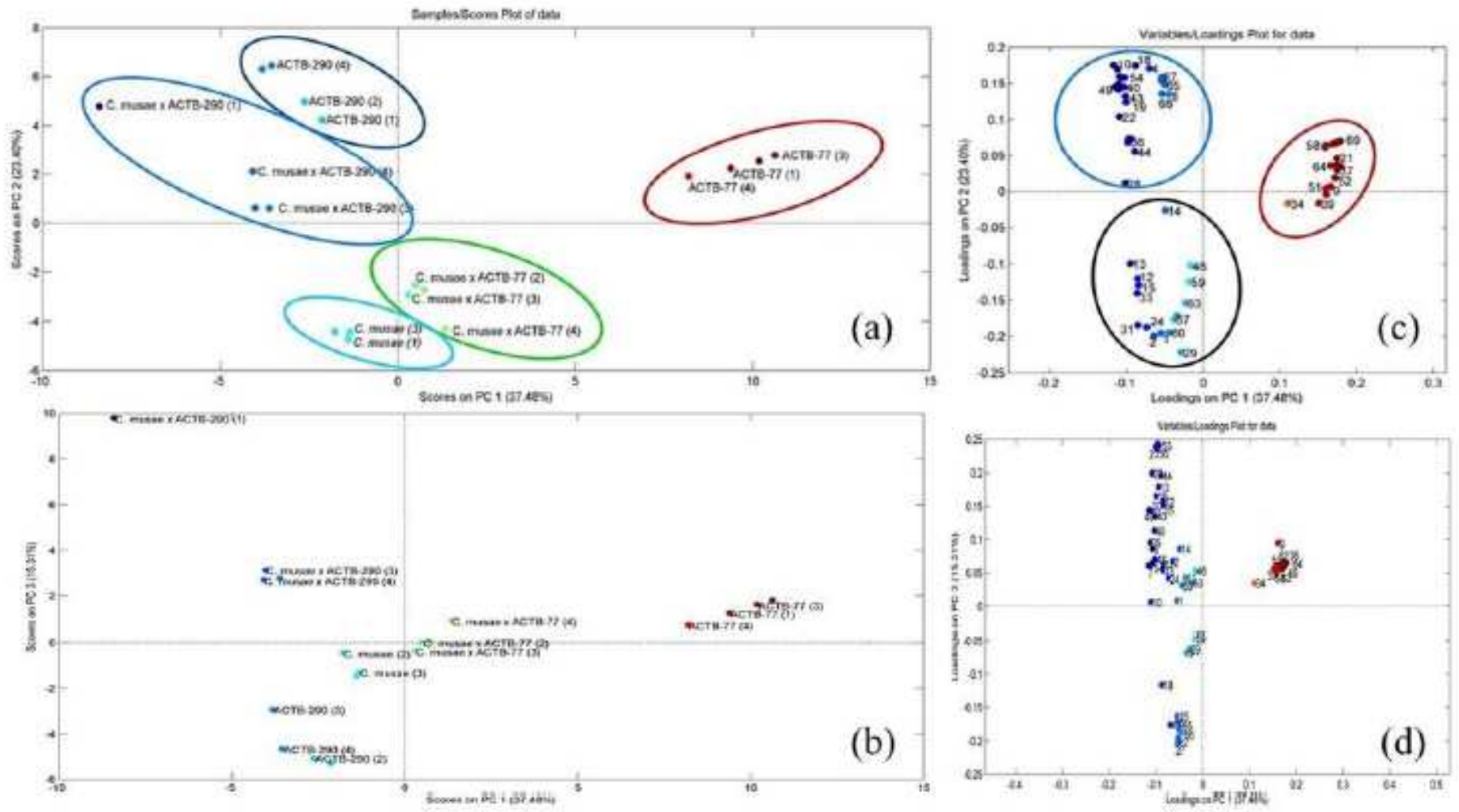

Figure 8

Scores (a) and loadings (b) graphs obtained by PC1 and PC2 for the samples ACTB-77 (dark red), ACTB290 (dark blue), C. musae (cyan) and co-cultures ACTB-77/C. musae (green) and ACTB-290/C. musae (light blue). 


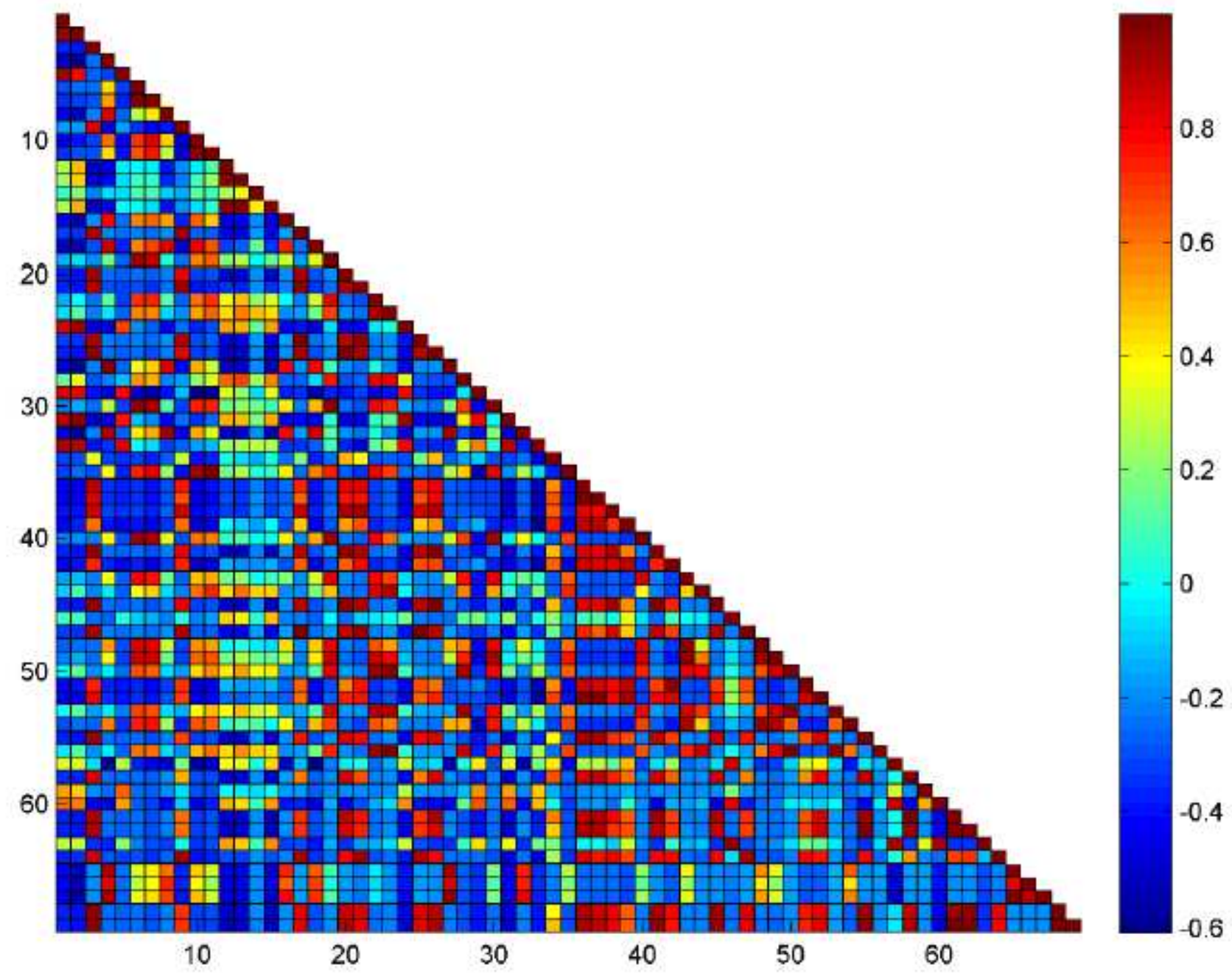

Figure 9

Correlation map for VOCs of the samples ACTB-77, ACTB-290, C. musae and co-cultures ACTB-77/C. musae and ACTB-290/C. musae. The correlation goes from -1 (dark blue) to 1 (dark red) scale and it was calculated pair by pair.

\section{Supplementary Files}

This is a list of supplementary files associated with this preprint. Click to download.

- SUPPLEMENTARYMATERIALVieiraetal.pdf 